Ann. Sci. forest., 1979, 36 (1), 59-92.

\title{
Premiers résultats d'une expérience d'élagage du peuplier I 214
}

\author{
R. KELLER \\ Station de Recherches sur la Qualité des Bois \\ Centre National de Recherches Forestières, I.N.R.A., \\ Champenoux, 54280 Seichamps
}

\begin{abstract}
Résumé
Des peupliers | 214 âgés de 7 ans ont subi des élagages de différentes intensités : les branches basses ont été coupées jusqu'à une hauteur proportionnelle à la hauteur totale des arbres. Des taux de $35,45,50,55,60$ et 65 p. 100 de cette hauteur ont été adoptés. Après 7 ou 8 saisons de végétation, les principaux résultats sont les suivants :

- L'élagage améliore la forme des troncs en provoquant la diminution de leur défilement : l'effet est plus marqué avec les taux d'élagage les plus élevés.

- La croissance en hauteur n'est ralentie dans aucun cas par l'élagage.

- L'élagage provoque un ralentissement de l'accroisseme t en circonférence dans la partie inférieure des troncs. Au voisinage de la base de la cime, le ralentissement est moins marqué. Seuls les taux les plus élevés, 60 p. 100 et 65 p. 100, ont un effet dépressif marqué sur tout le fronc.

- La production en volume est faiblement diminuée par l'élagage ; seuls les taux de 60 p. 100 et 65 p. 100 conduisent à une perte sensible de production. Cette perte est d'ailleurs compensée partiellement au débit par la meilleure forme des troncs qui est plus cylindrique.

- Pour des conditions de milieu comparables à celles de cette expérience, on peut recommander de pratiquer un élagage sur 50 à 55 p. 100 de la hauteur totale des arbres ; l'élagage doit commencer tôt et suivre régulièrement l'évolution des arbres. Dans le cas de 1214, il doit être complété par des défourchages et formations de cimes dès les premières années qui suivent la plantation.

- Il faudra à l'avenir examiner l'influence de l'intensité d'élagage sur la formation de bois de tension et l'apparition éventuelle d'un faux bois de cceur.
\end{abstract}

\section{1. - Introduction}

Une expérience d'élagage sur des peupliers I 214 installés en forêt de Retz a été entreprise à partir de 1968 par la Station de Recherches sur la Qualité des Bois. Le but en était d'étudier l'influence du taux d'élagage sur la qualité du bois produit, qu'elle résulte de la morphologie des arbres ou des propriétés physiques ou densitométriques de leur bois. Après une dizaine de saisons de végétation depuis les premiers élagages, il est possible d'examiner un certain nombre de résultats portant essentiellement sur la qualité résultant de la forme des arbres. 


\section{2. - Matériel et méthode}

\section{1. - Installation du dispositif et traitements}

En 1968, nous avons pu implanter en parcelle 7 de sixième série de la forêt de Retz un dispositif d'élagage sur des peupliers 1214 plantés, pour une partie au printemps 1962, et pour l'autre partie au printemps 1963. Le terrain présente un sol limo-

\section{TABLEAU 1}

Chronologie des opérations effectuées dans les dispositifs (I) et (II)

Working chronology in the plots (I) and (II)

\begin{tabular}{l}
\hline \\
\hline Printemps 1962 \\
(Spring 1962) \\
Printemps 1963 \\
(Spring 1963)
\end{tabular}

\section{3 à 1967}

Printemps 1968 (Spring 1968)

Printemps 1969 (Spring 1969)

Automne 1970 (Autumn 1970)

Automne 1971

(Autumn 1971)

Automne 1972

(Autumn 1972)

Printemps 1974 (Spring 1974)
Plantation (Planting) plot (I). latin square

Elagage partiel, défourchage (Partial pruning, cutting of multiple leaders)

Elagage à 50 p. 100,60 p. 100 de la hauteur totale. Témoins (Pruning up to 50 p. 100. 60 p. 100 tree height. Control plot)

Elagage à 35 p. 100,45 p. 100,55 p. 100 65 p. 100 de la hauteur totale. Témoins (Pruning up to 50 p. 100,60 p. 100 tree height. Control plot)

Taille de grosses branches et de cimes multiples (Trimming of large branches and mul tiple leaders)

Taille partielle de branches et de cimes (Partial trimming of branches and mulfiple lea. ders)

Elagage réajusté à 50 p. 100 ef 60 p. 100 de la hauteur totale. Elagage des branches sèches des témoins correspondant à un taux d'environ 35 p. 100 (Pruning readjustement up to 50 p. 100 and 60 p. 100 tree height. Dry pruning in control plots corresponding to an intensity of about 35 p. 100)
Elagage réajusté à 35 p. 100,45 p. 100 55 p. 100 et 65 p. 100 de la houteur totale Elagage des branches sèches des témoin: correspondant à un taux d'enviror 35 p. 100 (Pruning readjustement uf to 35 p. 100,45 p. 100,55 p. 100 anc 65 p. 100 tree height. Dry prunning if control plots corresponding to an inten sity of about 35 p. 100) 
neux profond. L'écartement des arbres est $7 \mathrm{~m} \times 7 \mathrm{~m}$. Entre l'installation des peupliers et le début de l'expérience d'élagage, il s'est écoulé six saisons de végétation, durant lesquelles les plants ont été partiellement élagués et défourchés.

Au début de 1968, nous avons installé, dans la partie plantée en 1962, une placette comportant trois traitements répartis dans un carré latin. Actuellement, deux cent cinquante-sept arbres au total y sont suivis, ce qui représente en moyenne environ vingt-huit arbres dans chacune des neuf parcelles unitaires de ce dispositif (I). Deux taux d'élagage y sont pratiqués :

- I'un jusqu'à une hauteur de 50 p. 100 de la hauteur totale de l'arbre,

- l'autre jusqu'à 60 p. 100.

Un traitement témoin oủ ne sont coupées que les branches sèches sert de référence.

L'année suivante, dans la partie plantée en 1963, une autre disposition fut choisie: sur des lignes de plantation, cinq modalités affectent cinq arbres successifs et ceci fut, à l'origine, répété trente-huit fois. Les taux adoptés sont 35 p. 100, 45 p. 100, 55 p. 100 et 65 p. 100 de la hauteur totale des arbres, ainsi qu'un traitement témoin où ne sont coupées que les branches sèches. Actuellement, il reste cent quatre-vingt-cing arbres et, par suite de quelques remaniements du schéma initial, le nombre de peupliers par traitement varie de trente-six à trente-neuf dans ce dispositif (II).

Le tableau 1 indique les diverses opérations pratiquées dans ces deux dispositifs:

Entre les opérations d'élagage furent effectués assez régulièrement dans le houppier des tailles de branches et des défourchages afin d'améliorer progressivement la forme de ce peuplier qui a tendance à être sinueux et à fourcher (Bonduelle P., 1971), et les gourmands ont été émondés.

Malheureusement, le début de cette expérience d'élagage n'a eu lieu que six années après l'installation des plants, et ceux-ci, bien qu'élagués et défourchés partiellement, mais pas chaque année, avaient déjà acquis souvent une forme défectueuse et de nombreuses grosses branches et fourches avaient eu le femps de se développer.

Les témoins ont subi également ces tailles et défourchages.

Les premiers élagages ont été effectués depuis le sol à l'aide d'un échenilloir ou d'une scie emmanchée déjà décrite dans un autre article (Polge H., 1967). Cet outillage convient jusqu'à des hauteurs d'environ $8 \mathrm{~m}$, à la rigueur $10 \mathrm{~m}$. Pour les besoins de l'expérience, nous avons élagué au-delà ; il a fallu alors procéder à la scie égoīne ou à la fronçonneuse à partir d'échelles du type « Ecole Forestière ».

\section{2. - Mesures}

Les mesures suivantes ont été effectuées pour chaque année de 1967 ou 1968 à 1975 (1974 exceptée) :

- hauteur totale initiale,

- longueur de la pousse annuelle,

- circonférence des troncs à $0,20 \mathrm{~m}$, à $1,30 \mathrm{~m}$, ainsi qu'à des hauteurs correspondant aux $1 / 3$ ef $2 / 3$ de la hauteur totale initiale pour les deux dispositifs. On a mesuré aussi la circonférence à la moitié de la hauteur initiale dans le dispositif (II). Lorsque les arbres ont afteint des dimensions suffisantes, une cinquième circonférence a été mesurée au niveau de cette hauteur initiale. 


\section{3. - Calculs}

Pour caractériser la forme des arbres, on a calculé le défilement, d'une part entre $1,30 \mathrm{~m}$ et les $2 / 3$ de la hauteur initiale, d'autre part entre $1,30 \mathrm{~m}$ et le niveau correspondant à la hauteur initiale à l'installation. Rappelons que le défilement est défini par :

$$
\frac{\mathrm{C}_{1}-\mathrm{C}_{2}}{\mathrm{~h}_{2}-\mathrm{h}_{1}}
$$

si $h_{1}$ et $h_{2}$ représentent deux niveaux de la grume $\left(h_{2}>h_{1}\right)$ dont les circonférences sont $C_{1}$ et $C_{2}$.

II exprime la décroissance en centimètres de la circonférence lorsqu'on passe d'un niveau de mesure à un niveau supérieur distant de $1 \mathrm{~m}$. II caractérise la forme des grumes, et plus il est faible, plus le tronc est cylindrique et meilleure est la forme.

Les calculs des volumes ont été effectués, pour des billes de pied de $6 \mathrm{~m}$ et de $8 \mathrm{~m}$, en utilisant ces défilements et en assimilant les portions de grumes à des troncs de cône.

\section{3. - Résultats}

\section{1. - Etat initial}

3.11. Haufeur totale moyenne.

- Dans le dispositif (I), la hauteur totale par parcelle unitaire variait à la fin de 1967 de $9,4 \mathrm{~m}$ pour une des parcelles unitaires du futur traitement à 60 p. 100 , à $10,2 \mathrm{~m}$ pour une des parcelles unitaires du futur traitement témoin, mais les différences entre futurs traitements n'étaient pas significatives.

- Dans le dispositif (II), elle variait à la fin de 1968 de $9,5 \mathrm{~m}$ (futur traitement à 65 p. 100 ) à $9,8 \mathrm{~m}$ (futur traitement à 55 p. 100) sans qu'il existe de différences significatives.

3.12. Circonférence moyenne à $1,30 \mathrm{~m}$.

- Dans le dispositif (I), la circonférence variait de $34,1 \mathrm{~cm}$ (futur traitement à 60 p. 100) d̀ $35,6 \mathrm{~cm}$ (futur traitement témoin).

- Dans le dispositif (II), elle allait de $35,2 \mathrm{~cm}$ (futur traitement à 65 p. 100) à $36,6 \mathrm{~cm}$ (futur traitement à 55 p. 100).

Aucune différence significative n'existait dans les deux dispositifs pour ce caractère.

\subsection{Circonférence moyenne à $6 \mathrm{~m}$.}

Du fait que les arbres peuvent avoir des hauteurs assez différentes, les circonférences mesurées à des fractions de ces hauteurs sont à des niveaux différents. Pour avoir des valeurs de circonférences plus comparables, on a estimé, à partir du défile- 
ment de la circonférence mesurée aux $2 / 3$ de la hauteur totale initiale, la valeur à $6 \mathrm{~m}$ de la circonférence de la bille de pied.

Dans le dispositif (I), celle-ci variait de $16,1 \mathrm{~cm}$ (futur traitement d̀ $60 \mathrm{p} .100$ ) d̀ $17,2 \mathrm{~cm}$ (futur traitement témoin).

Dans le dispositif (II), les bornes étaient $16,2 \mathrm{~cm}$ (futur traitement à $65 \mathrm{p} .100$ ) et $17,0 \mathrm{~cm}$ (futur traitement à 55 p. 100).

Tout comme pour la circonférence à $1,30 \mathrm{~m}$, aucune différence significative n'existait avant le début de l'élagage dans les deux dispositifs.

\subsection{Volume moyen entre $0 \mathrm{~m}$ et $6 \mathrm{~m}$.}

On a calculé ce volume en assimilant la bille de pied à un tronc de cône et en utilisant le défilement, la circonférence à $1,30 \mathrm{~m}$ et la circonférence à $6 \mathrm{~m}$. Il était compris, dans le dispositif (I) entre $0,039 \mathrm{~m}^{3}$ pour le futur traitement à 60 p. 100 et $0,043 \mathrm{~m}^{3}$ pour le futur traitement témoin. Dans le dispositif (II), ses valeurs étaient échelonnées entre $0,042 \mathrm{~m}^{3}$ (futurs traitements à 45 p. 100 et 65 p. 100 ) et $0,045 \mathrm{~m}^{3}$ (futur traitement à 55 p. 100). Pour cette caractéristique, il n'existait pas de différence significative à l'origine.

3.15. Défilement moyen entre $1,30 \mathrm{~m}$ et le niveau correspondant aux $2 / 3$ de la hauteur totale initiole.

Sa plus faible valeur, dans le dispositif (I), est $3,83 \mathrm{~cm} / \mathrm{m}$ (futurs traitements à 50 p. 100 et 60 p. 100 ) et sa plus forte, $3,92 \mathrm{~cm} / \mathrm{m}$, est celle du futur traitement témoin. Dans le dispositif (II), il va de $3,95 \mathrm{~cm} / \mathrm{m}$ (futur traitement à $45 \mathrm{p}, 100$ ), à $4,17 \mathrm{~cm} / \mathrm{m}$ (futur traitement à 55 p. 100).

Comme dans les cas précédents, aucune différence significative initiale n'était décelable.

3.16. Diamètre des premières branches vivantes.

On a mesuré dans le dispositif (II) de 1968, date du premier élagage, à 1973. juste avant le second, le diamètre des premières branches vivantes situées à la base de la cime. Le tableau 2 résume ces résultats. Quelle que soit l'année, l'analyse de variance aboutit à des tests $\mathrm{F}$ non significatifs. En particulier, en 1968, juste au moment du premier élagage et quel que soit le niveau de la cime, il n'y avait pas de différence dans les diamètres des premières branches vivantes.

\subsection{Remarques.}

Sans exception, tous les futurs traitements étaient comparables entre eux, que ce soit pour le dispositif (I) à la fin de 1967 ou le dispositif (II) à la fin de 1968 ; il semble toutefois que, sauf pour le défilement, le mode du choix des arbres dans celui-ci a conduit à une plus grande homogénéité des conditions de départ puisqu'on y observe entre les valeurs moyennes extrêmes des écarts de :

- 0,30 $\mathrm{m}$ pour la hauteur initiale contre $0,80 \mathrm{~m}$ pour le dispositif (I),

- 1,4 cm pour la circonférence à $1,30 \mathrm{~m}$ contre $1,5 \mathrm{~cm}$ pour le dispositif (I).

- $0,8 \mathrm{~cm}$ pour la circonférence à $6 \mathrm{~m}$ contre $1,1 \mathrm{~cm}$ pour le dispositif (I),

- $0,003 \mathrm{~m}^{3}$ pour le volume entre 0 et $6 \mathrm{~m}$ contre $0,004 \mathrm{~m}^{3}$ pour le dispositif (I). 


\section{TABLEAU 2}

Dispositif (II). Diamètre des premières branches vivantes à la partie inférieure de la cime (Plot (II). Diometer af the base of first living branches in the lower part of the crown)

\begin{tabular}{|c|c|c|c|c|c|c|c|}
\hline \multirow{2}{*}{$\begin{array}{c}\text { Traite- } \\
\text { ment } \\
\text { (treat- } \\
\text { ment) }\end{array}$} & \multirow{2}{*}{$\begin{array}{c}\text { Hauteur moyenne } \\
\text { en m des } \\
\text { branches mesu- } \\
\text { rées } \\
\text { (mean height in } \\
\text { metres above the } \\
\text { soil of the } \\
\text { measured } \\
\text { branches) }\end{array}$} & \multicolumn{6}{|c|}{ Diamètre moyen à la base en $\mathrm{mm}$} \\
\hline & & 1968 & 1969 & 1970 & 1971 & 1972 & 1973 \\
\hline $\begin{array}{l}\text { Témoins } \\
\text { (control } \\
\text { trees) }\end{array}$ & 3,65 & 19,6 & 21,2 & 25,8 & 30,3 & 30,4 & 35,3 \\
\hline 35 p. 100 & 3,70 & 19,8 & 21,3 & 25,3 & 29,8 & 30,3 & 34,5 \\
\hline 45 p. 100 & 4,40 & 18,9 & 20,7 & 25,2 & 28,9 & 29,4 & 33,3 \\
\hline 55 p. 100 & 5,40 & 20.4 & 22,5 & 27,1 & 31,4 & 31,5 & 35,7 \\
\hline 65 p. 100 & 6.20 & 17,9 & 20,0 & 23,9 & 28,0 & 29,3 & 34,2 \\
\hline Test F ... & - & N.S. & N.S. & N.S. & N.S. & N.S. & N. S. \\
\hline F test ... & & $\begin{array}{l}\text { test not si- } \\
\text { gnificant }\end{array}$ & $\begin{array}{l}\text { test not si- } \\
\text { gnificant }\end{array}$ & $\begin{array}{l}\text { test not si- } \\
\text { gnificant }\end{array}$ & $\begin{array}{c}\text { test not si- } \\
\text { gnificant }\end{array}$ & $\begin{array}{l}\text { test notsi- } \\
\text { gnificant }\end{array}$ & $\begin{array}{l}\text { test not si- } \\
\text { gnificant }\end{array}$ \\
\hline
\end{tabular}

Le défilement était toutefois plus homogène dans le dispositif (I) puisqu'on n'y observe qu'une différence de $0,09 \mathrm{~cm} / \mathrm{m}$ contre $0,22 \mathrm{~cm} / \mathrm{m}$ dans le dispositif (II).

Si la disposition en carré latin du dispositif (I) est plus démonstrative à l'œil puisque les parcelles unitaires regroupent un assez grand nombre d'arbres subissant le même traitement, en revanche, elle semble avoir conduit à une plus grande hétérogénéité des conditions de départ, sans toutefois avoir provoqué l'apparition de différences significatives.

\section{2. - Evolution des caractéristiques après l'élagage}

\subsection{Hauteur totale.}

Dans le dispositif (I), en 1973, soit six années après le premier élagage défini dans cette expérience et une année après le second, on trouve des différences de hauteur entre traitements significatives au seuil de 5 p. 100 ; les témoins ef le traitement 50 p. 100 sont en première place avec respectivement $16,7 \mathrm{~m}$ et $16,6 \mathrm{~m}$ et se distinguent des arbres traités à 60 p. 100 avec $16,3 \mathrm{~m}$ (tabl. 3). II faut rappeler que dans l'état initial, les témoins avaient en moyenne $0,10 \mathrm{~m}$ de plus que les futurs arbres traités d 50 p. 100 et $0,20 \mathrm{~m}$ de plus que les futurs arbres traités à 60 p. 100 . L'écart s'est donc seulement légèrement creusé entre témoins et 50 p. 100 d'une part, et 60 p. 100 d'autre part, ce qui a suffi pour faire apparaitre des différences significatives. Deux ans plus tard, le classement restait le même, avec respectivement $19,8 \mathrm{~m}, 19,7 \mathrm{~m}$ et $19,4 \mathrm{~m}$. 


\section{TABLEAU 3}

Dispositif $(I)$, carré latin. Tests de signification des comparaisons de moyennes entre les différents traifements

Plof (I), latin square. Signification fests of comparisons of means between the several treatments

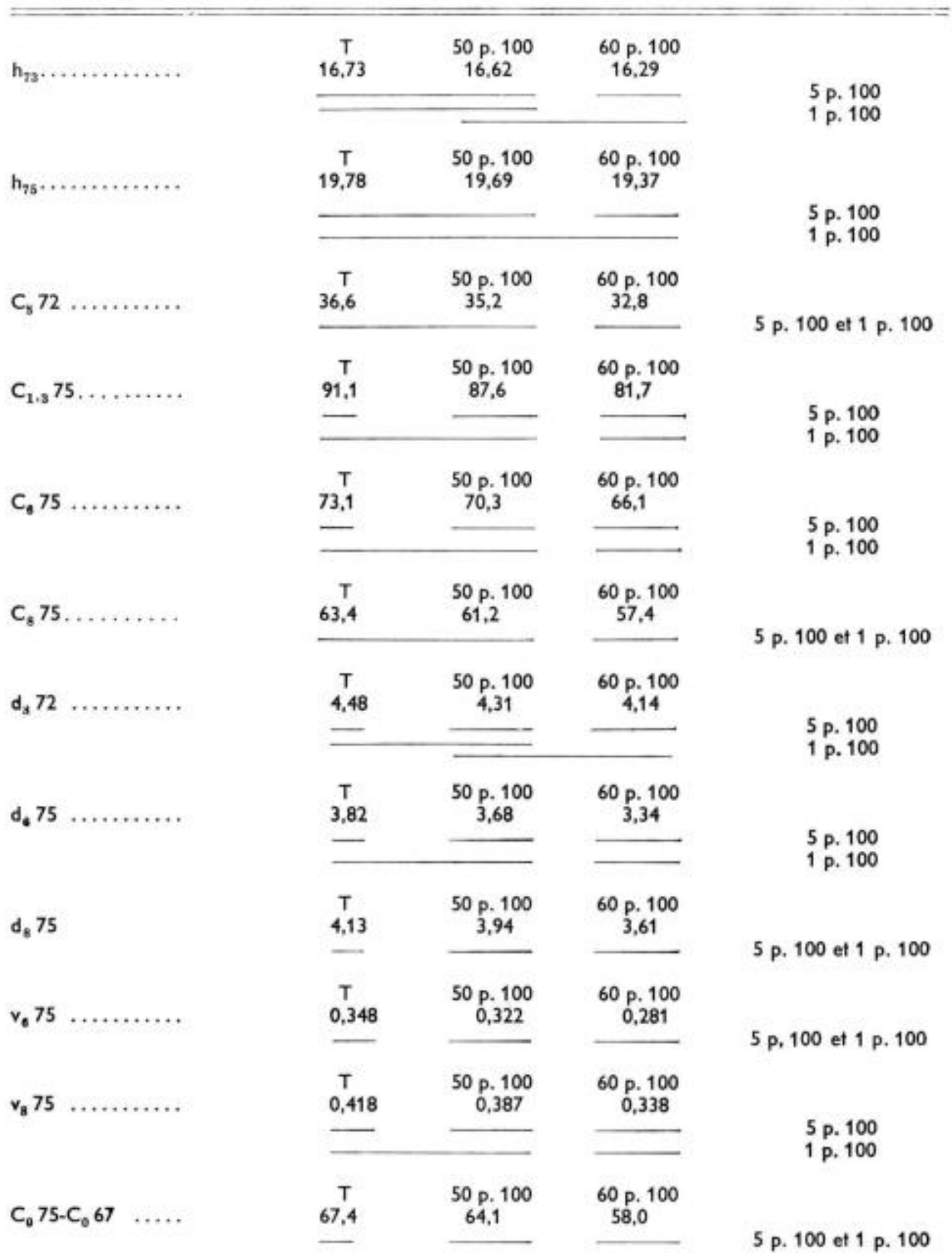




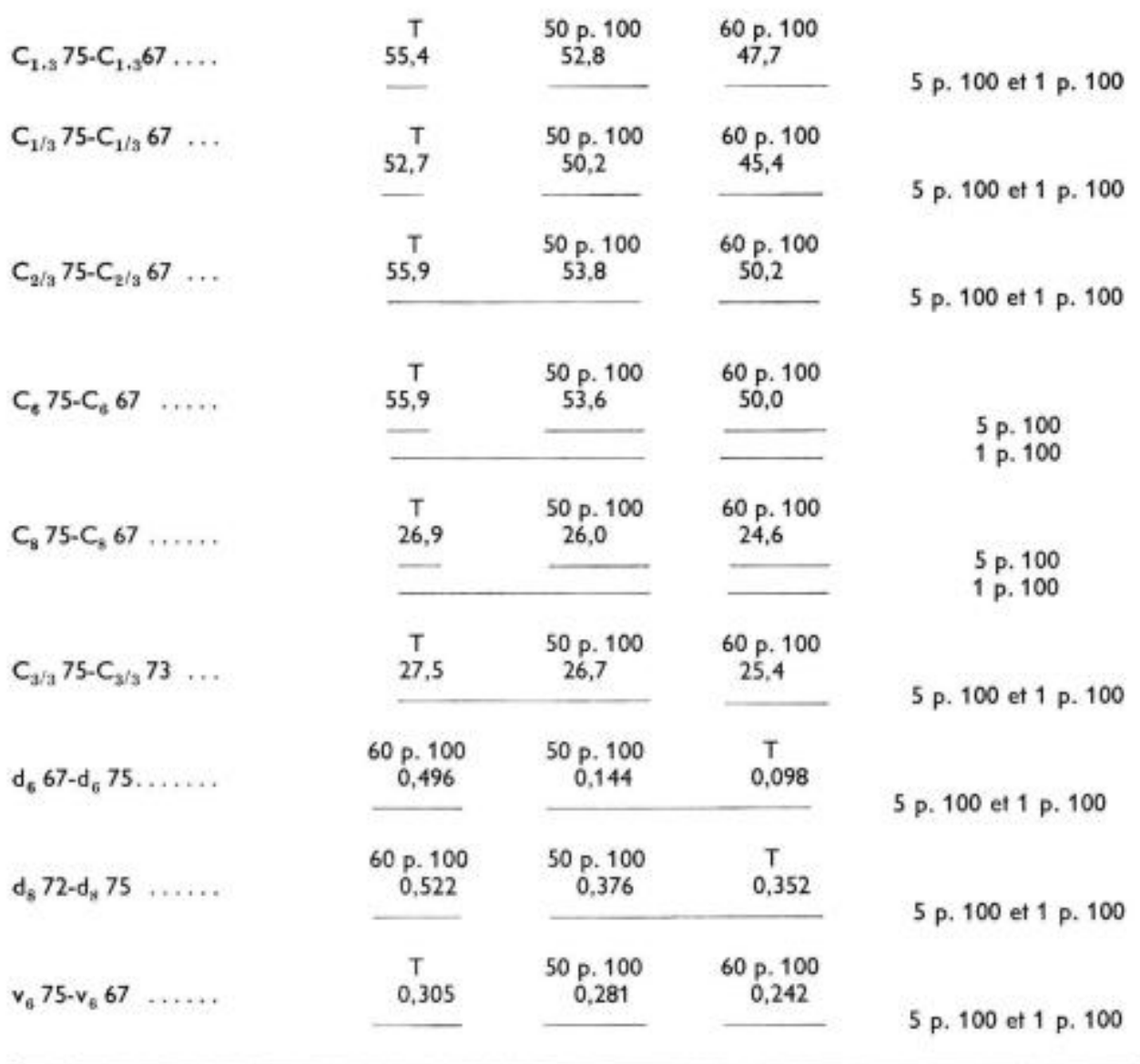

Signification des abrériations. Exemples (Abbreviations meaning. Examples.)

$h_{23}=$ hauteur totale à la fin de l'année 1973 (total height at the end of 1973).

C. 72 - circonférence à $8 \mathrm{~m}$ d̀ la fin do l'anné 1972 (circumference at $8 \mathrm{~m}$ level (in cm) at the end of 1972 ).

d, 72 = défilement entre $1,30 \mathrm{~m}$ et $8 \mathrm{~m}$ à la fin de l'année 1972 (circumference taper (in cm/ $\mathrm{m}$ ) between $1,30 \mathrm{~m}$ and $8 \mathrm{~m}$ levels at the end of 1972 ).

$v_{5} 75=$ volume entre $0 \mathrm{~m}$ et $6 \mathrm{~m}$ da la fin de l'année 1975 (volume in $\mathrm{m}^{3}$ ) between $0 \mathrm{~m}$ and $6 \mathrm{~m}$ levels at the end of 1975).

$c_{1}, 75=$ circonférence au $1 / 3$ de la hauteur totale initiale à la fin de l'année 1975 (circumference fin cm) a the third of the initial tree height, at the end of 1975).

5 p 100, 1 p. $100=$ significant differences between treatments at the 5 p. 100,1 p. 100 levels ; several treatments underfined by the same stroke are not statistically different of each others.

Dans le dispositif (II), en 1973, soit cinq années après le premier élagage et juste avant le second, aucune différence significative n'est apparue dans les hauteurs, les valeurs moyennes extrêmes étant données par les traitements témoins, 35 p. 100 et 55 p. 100 avec $15,8 \mathrm{~m}$ et par le traitement 65 p. 100 avec $15,6 \mathrm{~m}$. Le traitement 55 p. 100 était déjà le plus grand à l'installation avec $9,8 \mathrm{~m}$ et le traitement $65 \mathrm{p}$. 100 le plus petit, avec $9,5 \mathrm{~m}$. La situation à la fin de 1975 ne laisse apparaître qu'une légère évolution dans le classement : le traitement 55 p. 100 reste en tête avec $19,4 \mathrm{~m}$, les témoins, 35 p. 100,45 p. 100 et 55 p. 100 rejoignant le traitement 65 p. 100 avec $19,1 \mathrm{~m}$. 


\subsection{Accroissement en hauteur.}

Si l'on examine, non plus les hauteurs, mais les accroissements en hauteur entre la fin de 1967 et la fin de 1975, toute différence significative disparaît, l'écart moyen entre résultats extrêmes n'atteignant que $0,2 \mathrm{~m}$ puisque le traitement à $50 \mathrm{p} .100 \mathrm{a}$ poussé de $10 \mathrm{~m}$ environ, contre $9,8 \mathrm{~m}$ pour le traitement à $60 \mathrm{p} .100$.

Bien que les accroissements en hauteur entre la fin de 1968 et la fin de 1975 ne montrent aucune différence significative entre traitements, on peut noter que ce sont les traitements 55 p. 100 et 65 p. 100 qui ont eu la progression la plus forte avec $9,6 \mathrm{~m}$, devant les autres qui ont poussé d'environ $9,4 \mathrm{~m}$.

L'examen des accroissements annuels en hauteur, en particulier les pousses de 1974 et 1975, n'a donc pas montré de différence entre les traitements dans les deux dispositifs.

P. Bonduelle (1971, op. cit.) avait pourtant observé que l'élagage de I 214 traités à partir de l'âge de 4 ans favorisait très nettement leur croissance en hauteur. Celle-ci apparaissait même comme proportionnelle à l'intensité d'élagage retenu.

Peut-être du fait que nos dispositifs ont été installés dans des peuplements plus âgés, nous n'avons pas observé un fait aussi net.

Pour le dispositif (I), on peut toutefois dire que l'élagage ne provoque pas de diminution de la croissance en hauteur.

Pour le dispositif (II), il est possible qu'une tendance se manifeste : au taux les plus élevés correspondent une croissance en hauteur un peu plus forte ; si elle se maintient, les différences observées deviendront peut-être significatives dans les années à venir.

\subsection{Circonférence à $1,30 \mathrm{~m}$.}

Dans le dispositif (I), les mesures faites à la fin de 1975 montrent que le traitement 60 p. 100 avec $81,7 \mathrm{~cm}$ est significativement inférieur aux traitements 50 p. $100(87,6 \mathrm{~cm})$ et témoin $(91,1 \mathrm{~cm})$ au seuil de 1 p. 100 ; au seuil de 5 p. 100 , les trois traitements peuvent même être considérés comme tous différents.

Dans le dispositif (II), le traitement à 65 p. 100 se sépare très nettement de l'ensemble des autres traitements au seuil de 1 p. 100 ; avec $80,2 \mathrm{~cm}$, sa circonférence est inférieure d'au moins $6 \mathrm{~cm}$ à celles des autres modalités (tabl. 4).

\subsection{Accroissement de la circonférence d̀ $1,30 \mathrm{~m}$.}

Si l'on considère les figures 1 et 2 , on se rend compte que, dès la première saison de végétation qui suit l'élagage, les traitements s'écartent des témoins. Dans le dispositif (I), ils s'ordonnent de façon contraire à l'intensité des taux et le traitement 60 p. 100 s'éloigne progressivement et régulièrement des deux autres.

L'accroissement sur cette circonférence entre la fin de 1967 et la fin de 1975 aboutit à des résultats sensiblement différents puisqu'ils sont disjoints même au seuil de 1 p. 100 : les témoins viennent en premier avec $55,4 \mathrm{~cm}$, suivis du traitement $50 \mathrm{p}$. 100 avec $52,8 \mathrm{~cm}$ et du traitement 60 p. 100 avec $47,7 \mathrm{~cm}$.

Dans le dispositif (II), les témoins et les traitements 35 p. 100 et 45 p. 100 restent pour ainsi dire constamment identiques ; bien qu'encore très proche, le traitement 55 p. 100 s'en écarte plus nettement dès la première saison qui suit l'opération, le 
TABLEAU 4

Dispositif (II), en lignes. Tests de signification des comparaisans de moyennes entre les différents traitements

(Plot (II), in rows. Signification tests of comparisons of means between the several treatments)

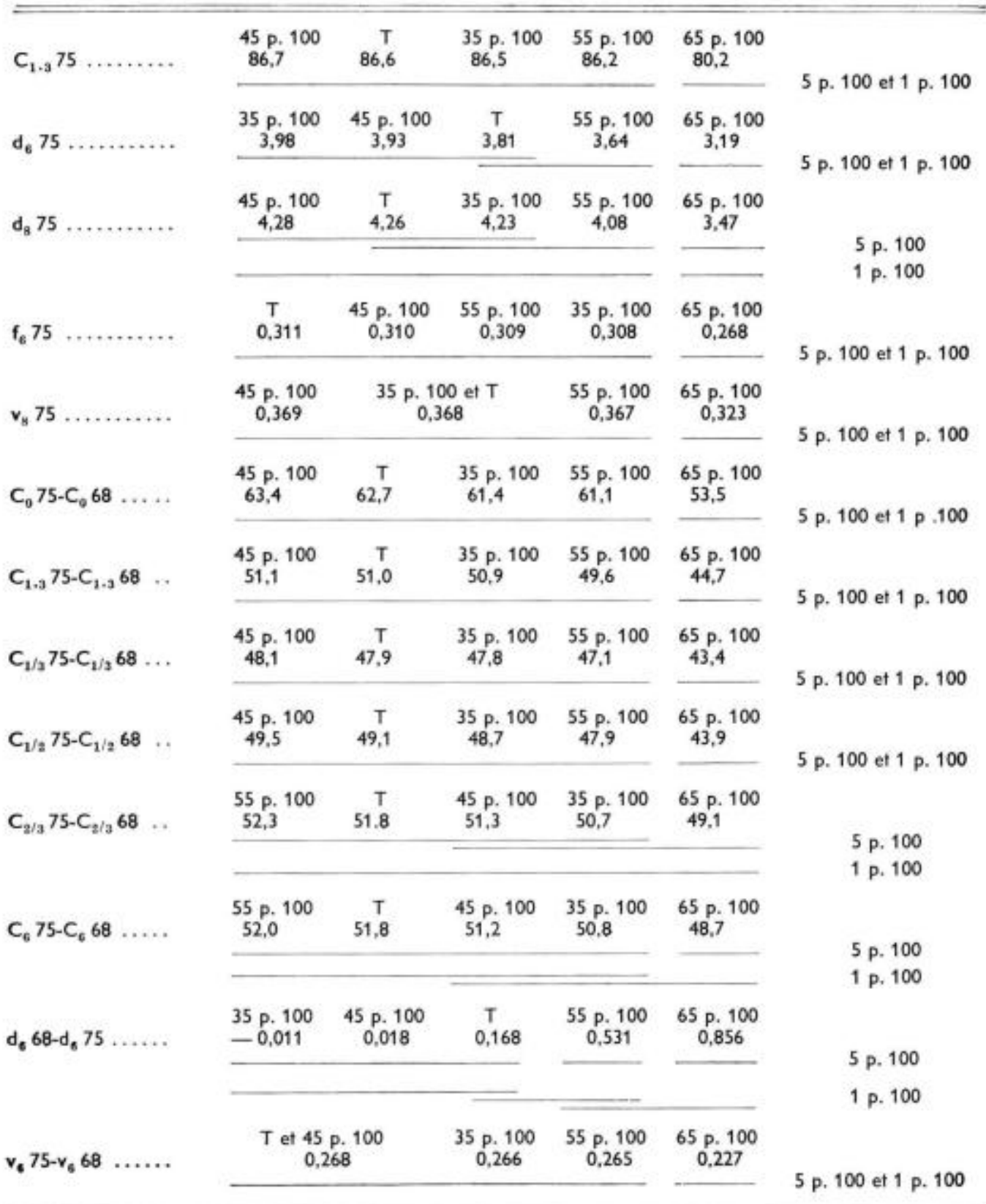


réajustement d'élagage aux taux initiaux fait après la saison de végétation de 1973 accusant légèrement le phénomène. Seul le troitement 65 p. 100 s'écarte franchement des autres pour donner la différence significative observée à la fin de 1975. Le réajustement d'élagage de 1973 semble avoir renforcé une évolution qui s'estompait, cinq saisons de végétation s'étant écoulées depuis le premier élagage.

\subsection{Circonférences à $6 \mathrm{~m}$ et $8 \mathrm{~m}$.}

La circonférence à $6 \mathrm{~m}$ dans le dispositif (I) donne une différence significative entre les traitements au seuil de 1 p. 100 , les témoins atteignant $73,1 \mathrm{~cm}$ en 1975 contre $66,1 \mathrm{~cm}$ pour l'élagage à 60 p. 100 . Le traitement 50 p. 100 a une valeur intermédiaire se rapprochant plus des témoins, la différence avec ceux-ci, observée au seuil de 5 p. 100, disparaissant au seuil de 1 p. 100.
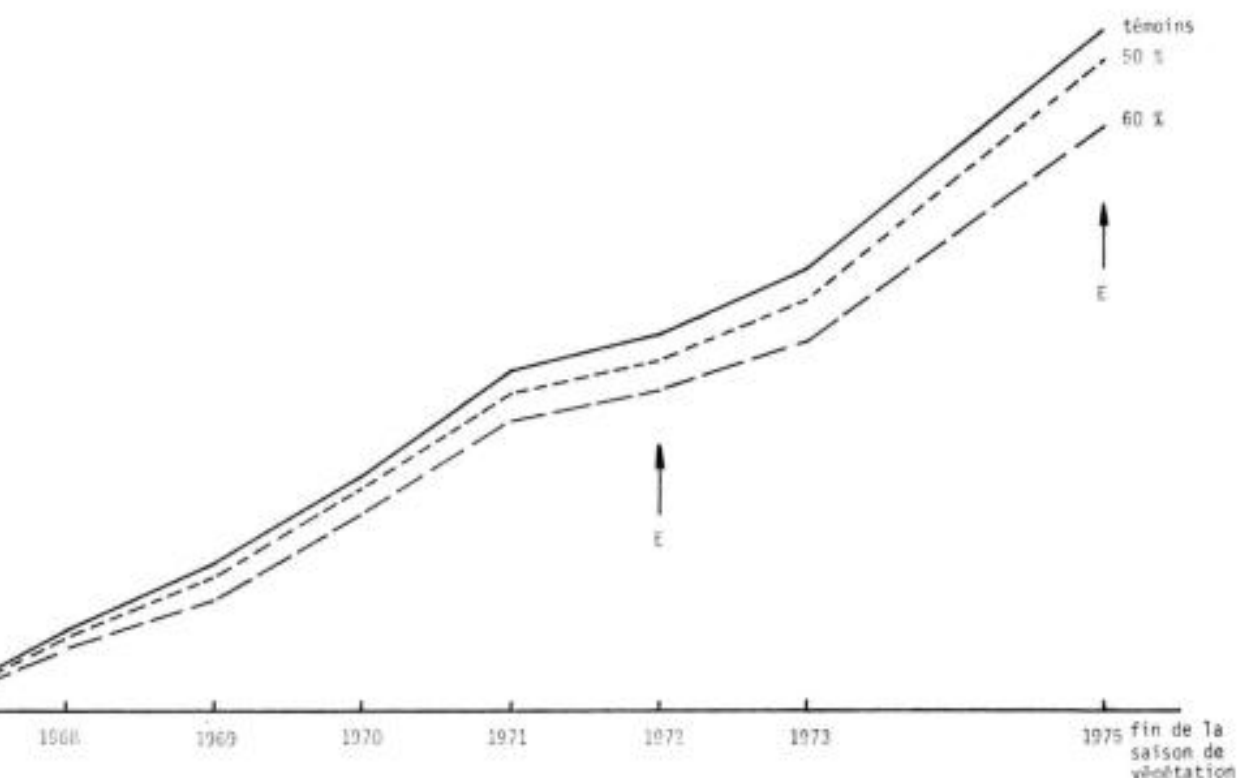

FIG. 1. - Villers-Cotterets. Carré latin. Accroissement de la circonférence à 1,30 m depuis la fin de 1967.

Fin de la saison de végétation.

Pour les graphiques 1 à 8 , la flêche associée à la lettre E signifie qu'il y a eu, à la date indiquée, élagage ou réélagage aux taux fixés pour l'expérience.

Villers-Cotterets, latin square. Girth increase at $1,30 \mathrm{~m}$ level from the end of 1967.

End of the vegetation season.

For the 1 to 8 graphes, arrow with $\mathbf{E}$ indicates that, at mentionned date, pruning or pruning readjustement was done at intensities fixed for the experimentation. 
Accroissenent de la. circonference 21,30 n

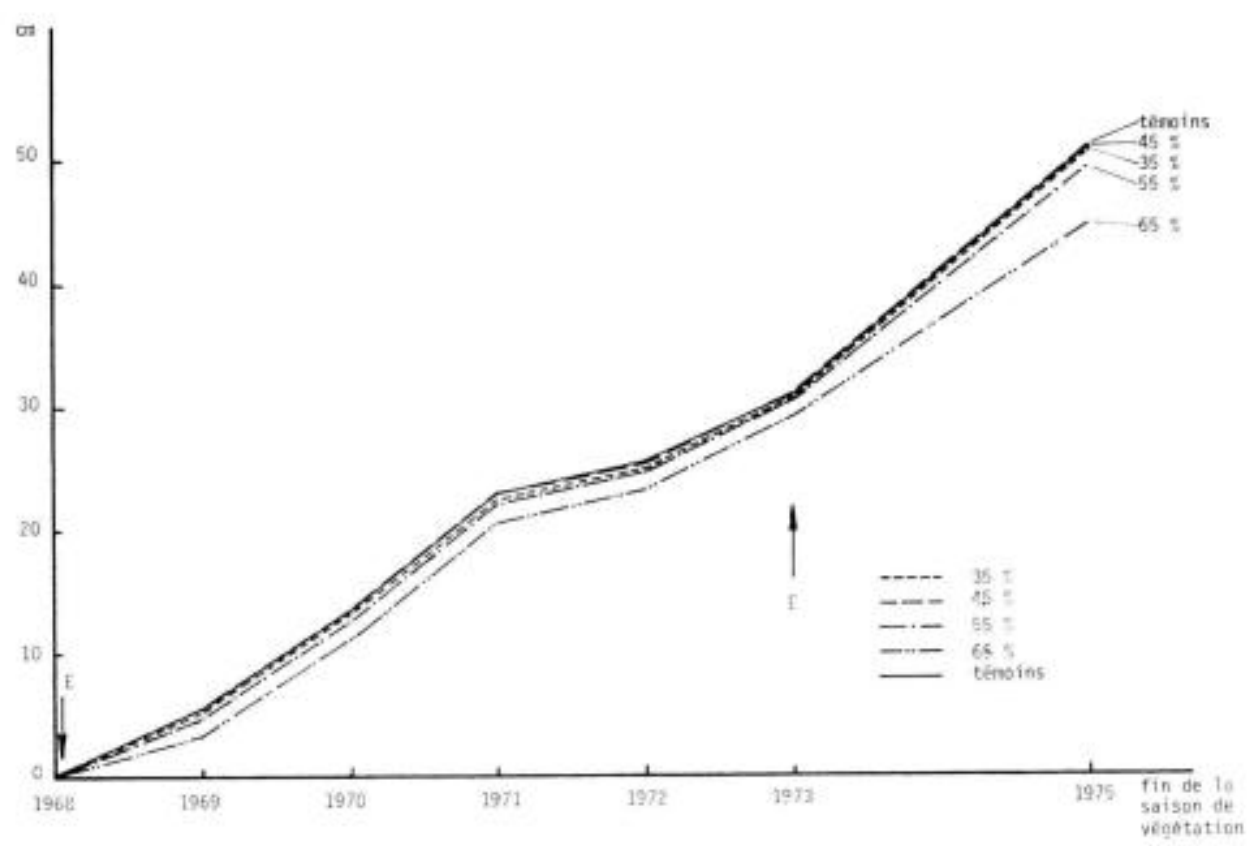

FIG. 2. - Villers-Cotferets. Dispositif en lignes. Accroissement de la circonférence à 1,30 m depuis la fin de 1968 .

Villers-Cotterets, rows. Girth increase at $1,30 \mathrm{~m}$ level from the end of 1968

La circonférence à $8 \mathrm{~m}$ calculée pour 1972 présentait déjà une différence significative au seuil de 1 p. 100 ; en 1975 , le traitement à 60 p. 100 s'est encore plus éloigné des témoins.

Dans le dispositif (II), aucun écart entre traitements ne se manifeste, que ce soit à $6 \mathrm{~m}$ ou à $8 \mathrm{~m}$.

\subsection{Accroissement sur les circonférences à $6 \mathrm{~m}$ ef $8 \mathrm{~m}$ et aux autres niveaux.}

Si l'on examine maintenant les accroissements de ces variables dans les deux dispositifs, on voit que seuls les deux traitements les plus forts se distinguent sensiblement de tous les autres.

Dans le dispositif ( 1 ), la circonférence à $6 \mathrm{~m}$ s'est accrue pour le traitement $60 \mathrm{p}$. 100 de $50,0 \mathrm{~cm}$ en huit saisons de végétation contre $55,9 \mathrm{~cm}$ pour les témoins ; entre 1972 et 1975 , la circonférence à $8 \mathrm{~m}$ a progressé de $24,6 \mathrm{~cm}$ chez les arbres élagués à 60 p. 100 contre $26,9 \mathrm{~cm}$ pour les témoins. II semble que pour les deux niveaux le traitement 50 p. 100 reste plus voisin du témoin que du traitement 60 p. 100 , la différence qui existe au seuil de 5 p. 100 disparait au seuil de 1 p. 100.

Dans le dispositif (II), pour le niveau $6 \mathrm{~m}$, le calcul montre que tous les traitements se sont comportés comme le témoin, avec des accroissements compris entre $52,0 \mathrm{~cm}$ et $50,8 \mathrm{~cm}$ pour sept saisons de végétation, sauf le traitement à 65 p. 100 qui se distingue significativement de ce groupe au seuil de 5 p. 100 avec $48,7 \mathrm{~cm}$. 
Sur les tableaux 2 et 3 sont également portés les accroissements en circonférence entre l'année d'installation de 1975, aux divers niveaux effectivement mesurés sur le terrain.

Pour le dispositif (I), on remarque que les trois traitements sont tous significativement différents les uns des autres pour la circonférence au pied de l'arbre d̀ environ $0,2 \mathrm{~m}$ ef pour la circonférence au $1 / 3$ de la hauteur initiale, soit environ $3,25 \mathrm{~m}$. Pour les valeurs des circonférences aux $2 / 3$ de la hauteur initiale, environ $6,45 \mathrm{~m}$, et à la hauteur initiale, environ $9,70 \mathrm{~m}$, les témoins et le traitement 50 p. 100 ne peuvent plus être considérés comme différents, seul le traitement 60 p. 100 reste bien à part.

Pour le dispositif (II), il n'existe aucune différence entre les accroissements en circonférence pour les traitements 35 p. 100, 45 p. 100, 55 p. 100 et témoins à quelque niveau que ce soit $(0,20 \mathrm{~m}, 3,25 \mathrm{~m}, 4,85 \mathrm{~m}$ et $6,45 \mathrm{~m})$. Le traitement $65 \mathrm{p} .100$ reste d part, sauf dans le cas de la circonférence aux $2 / 3$ de la hauteur initiale, où il se regroupe avec les traitements 35 p. 100 et 45 p. 100 au sevil de 5 p. 100, et avec tous les traitements au seuil de 1 p. 100.

Les graphiques des figures 3 et 4 , construits à partir des circonférences mesurées au niveau correspondant aux $2 / 3$ de la hauteur initiale des arbres, illustrent la croissance à environ $6,5 \mathrm{~m}$ en moyenne, donc à une hauteur voisine du niveau $6 \mathrm{~m}$.

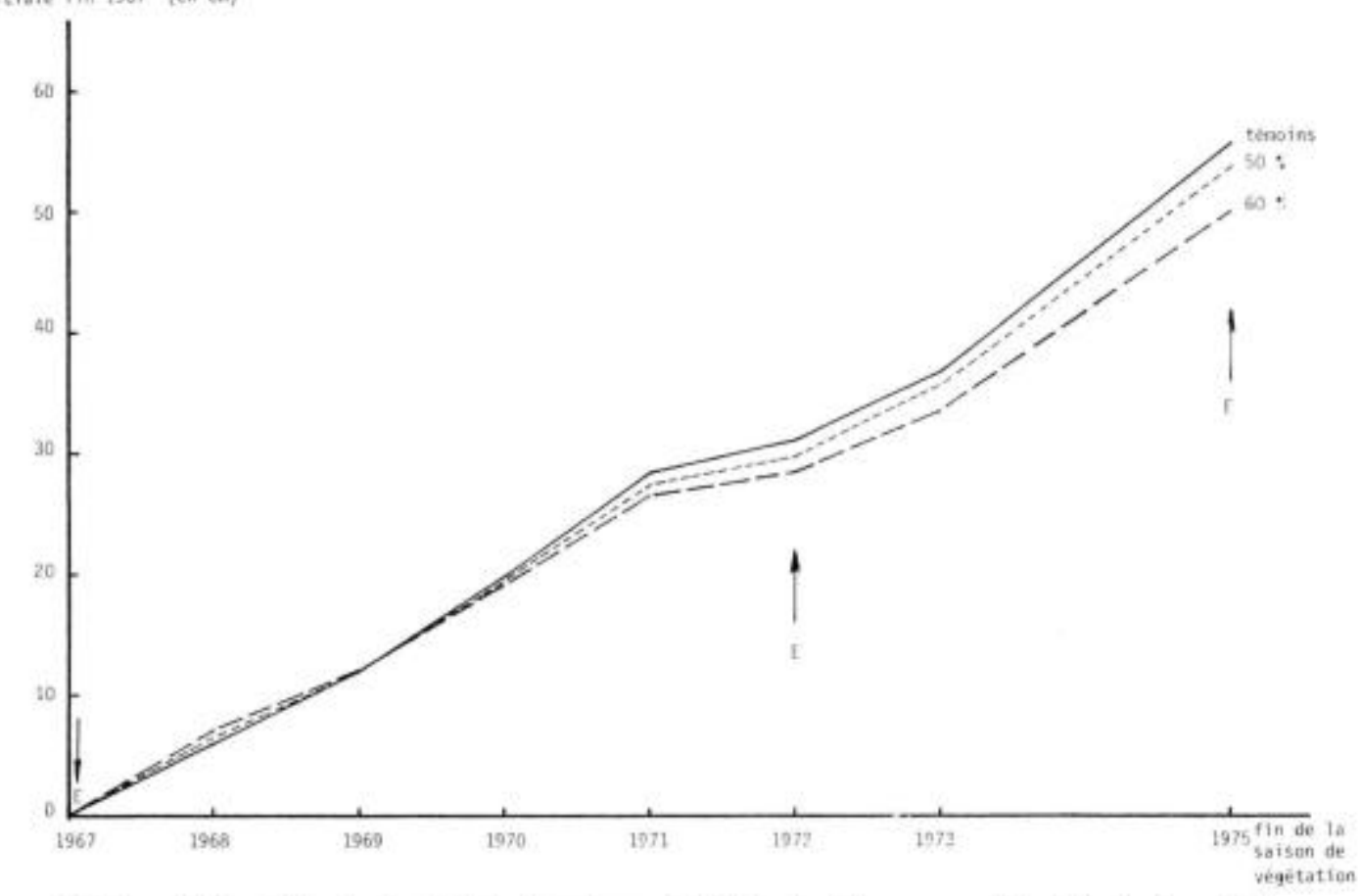

FIG. 3. - Villers-Cotterets. Carré latin. Accroissement de la circonférence aux $2 / 3$ de la hauteur totale initiale à la fin de 1967 (environ 6,50 m).

Villers-Cotterets, latin square. Girth increase of $2 / 3$ total initial height level at the end of 1967 


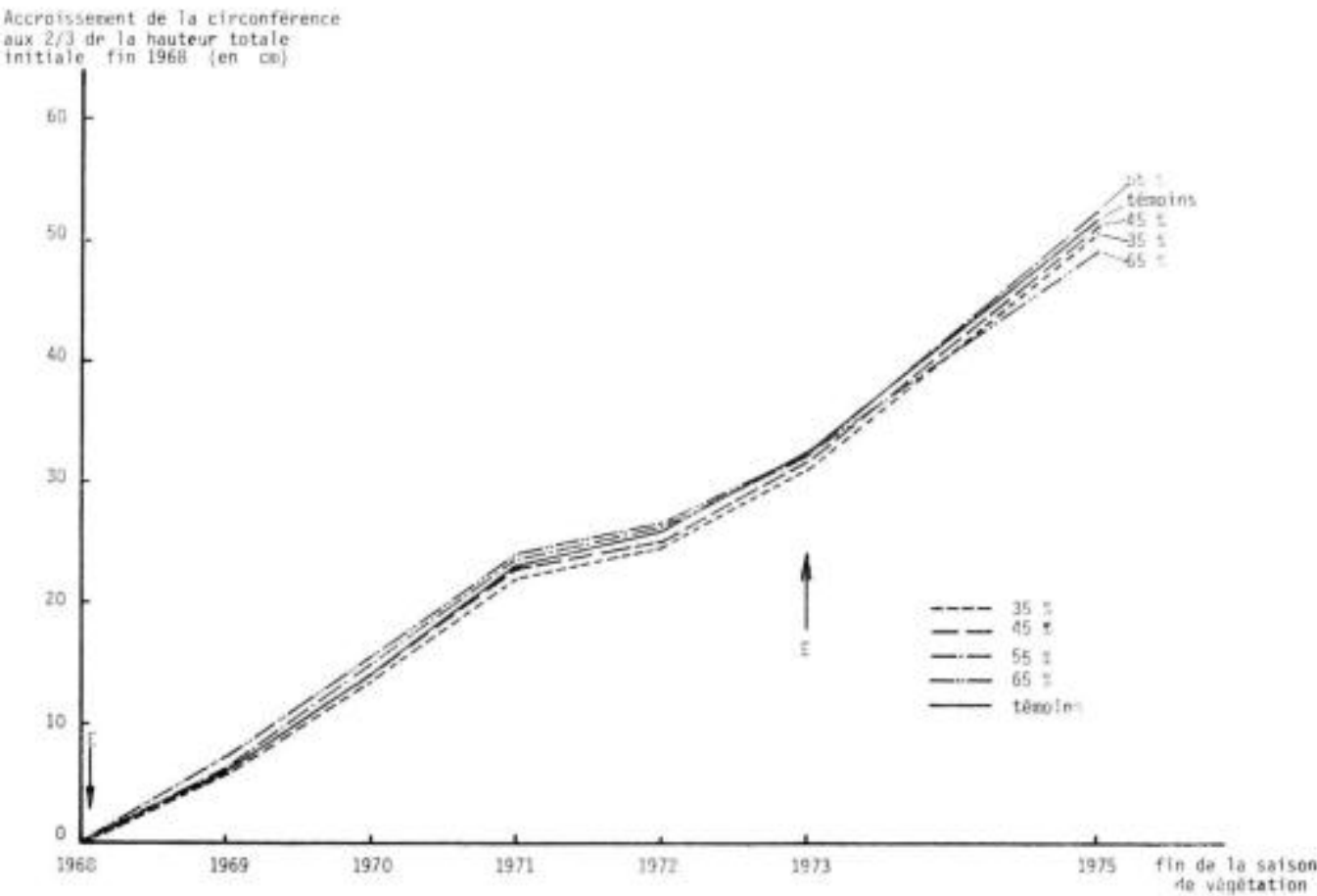

FIG. 4. - Villers-Cotferets. Dispositif en lignes. Accroissement de la circonférence aux 2/3 de la hauteur totale initiale da la fin de 1968 (environ 6,50 m).

Villers-Cotterefs, rows. Girth increase at 2/3 fotal initial height at the end of 1968 (about $6,50 \mathrm{~m}$ ).

Dans le dispositif (I), les traitements ne commencent à se séparer nettement qu'à la fin de 1971, soit quatre ans après le premier élagage; par la suite, le traitement 60 p. 100 s'écarte régulièrement des deux autres pour aboutir à des différences significatives que l'on a vues à propos du niveau $6 \mathrm{~m}$.

Dans le dispositif (II), le traitement 65 p. 100 a un accroissement supérieur à tous les traitements de 1969 à 1972, puis il ralentit nettement pour se trouver à la fin de 1975 significativement inférieur à tous les autres.

La comparaison des années communes des graphiques des deux dispositifs, aussi bien à $1,30 \mathrm{~m}$ qu'à $6,50 \mathrm{~m}$, semble montrer qu'un effet « année » se superpose à l'effet « élagage ». Les courbes du dispositif (II) présentent une évolution exactement comparable à celles du dispositif (I), tout en montrant une vitesse de croissance un peu plus forte. Pour les deux graphiques, les concavités vers le haut se trouvent en 1969, 1972 et 1973, alors que le dispositif (I) a été réélagué avant la saison végétation 1973. et le dispositif (II) avant celle de 1975.

Or ces concavités vers le haut correspondent à des accélérations de croissance en 1970, 1973 et 1974, et comme elles affectent tous les traitements, que ce soit une année d'élagage ou pas, il semble bien que l'effet « année » ait une grande influence sur la croissance, l'élagage intervenant diversement selon les taux sur la pente de ces graphiques. L'évolution des taux 60 p. 100 et 65 p. 100 après 1972 illustre cette 
constatation : ils suivent le schéma général de croissance, tout en devenant moins rapides. Une concavité vers le bas s'observe après 1971, indépendamment semble-t-il de tout effet d'élagage, puisqu'on se trouve à quatre années du premier élagage dans le dispositif (I) et à trois ans dans le dispositif (II). Elle traduit probablement que la saison de végétation de 1972 a été peu favorable à la croissance des peupliers.

II est curieux de constater, même si cela n'est pas significatif, que juste après le premier élagage dans les deux dispositifs, les traifements les plus forts s'accroissent plus vite que les témoins au niveau $6,5 \mathrm{~m}$ : c'est le cas des taux $50 \mathrm{p} .100$ et $60 \mathrm{p} .100$ du dispositif (I) en 1968 , et des taux 55 p. 100 et 65 p. 100 du dispositif (II) de 1969 à 1972.

Par la suite, ces traitements perdent du terrain par rapport aux arbres non élagués ou moins élagués, à l'exception du traitement 55 p. 100 qui, très voisin des témoins, s'est maintenu presque constamment au-dessus d'eux.

\subsection{Défilement.}

\subsection{Défilement entre $1,30 \mathrm{~m}$ et $6 \mathrm{~m}$.}

L'élagage a eu une action nette sur l'évolution du défilement entre $1,30 \mathrm{~m}$ et $6 \mathrm{~m}$. A l'origine, aucune différence significative n'existait entre les divers groupes d'arbres

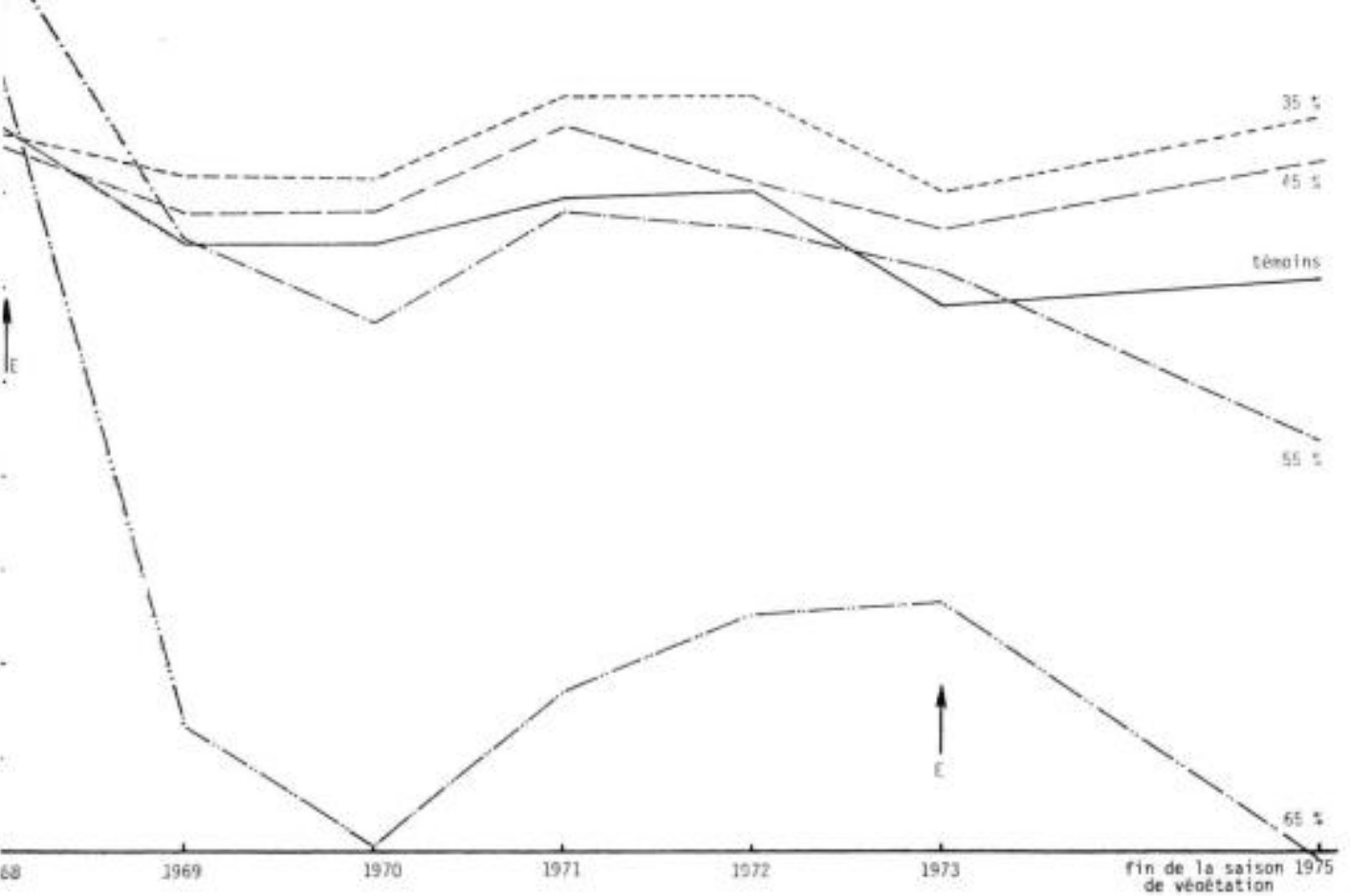

FIG. 5. - Villers-Cotterets. Dispositifs en ligne. Défilement entre 1,30 m et $6 \mathrm{~m}$. Villers-Colterefs, rows. Girth taper beiween 1,30 and $6 \mathrm{~m}$. 


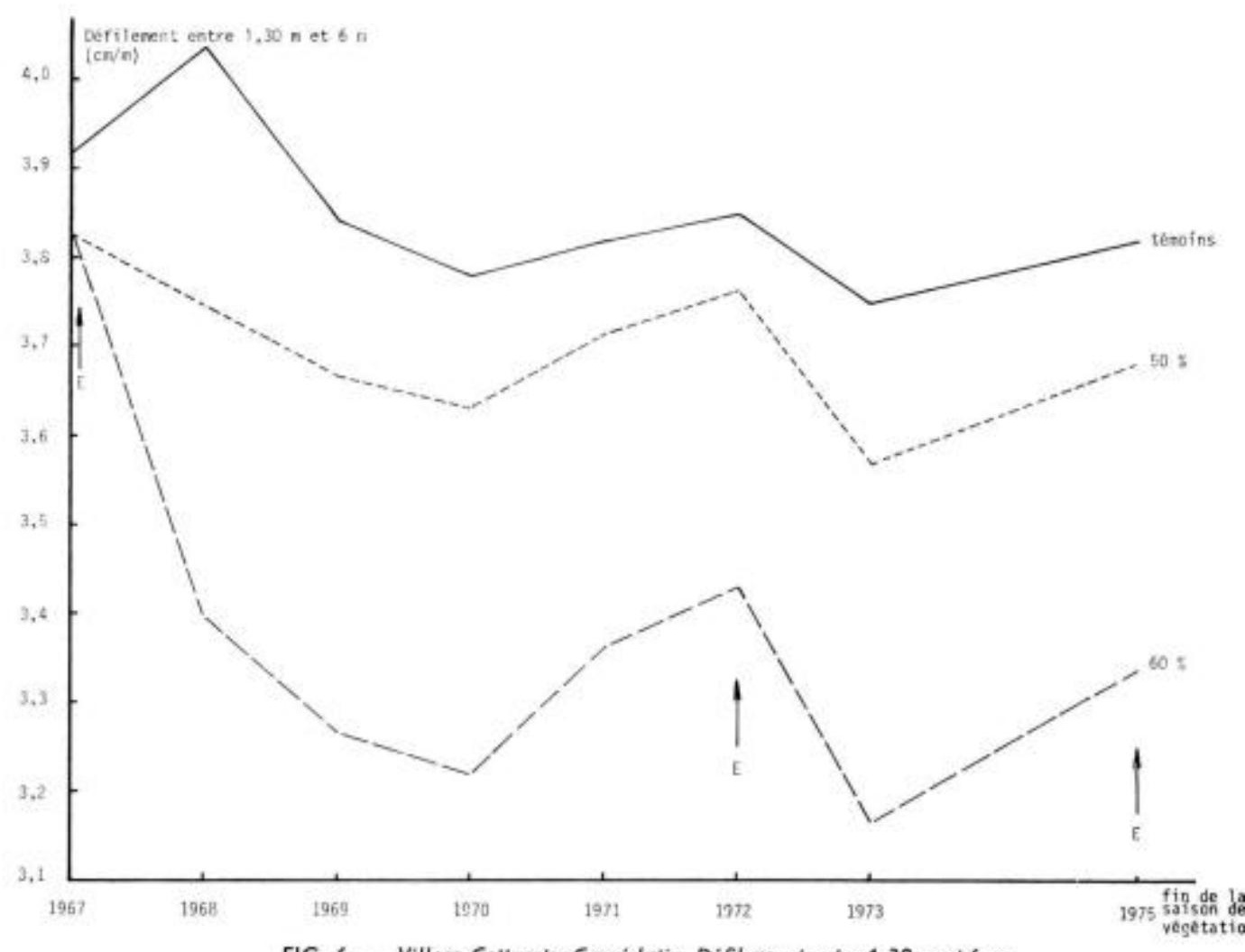

FIG. 6. - Villers-Cotterets. Carré Iatin. Défilement entre 1,30 $\mathrm{m}$ et $6 \mathrm{~m}$.

Villers-Cotterets, latin square. Girth taper between 1,30 and $6 \mathrm{~m}$.

destinés aux différents traitements. Dans la saison qui suit le premier élagage, les taux les plus forts, 50 p. 100 dans le dispositif (I), 55 p. 100 et 65 p. 100 dans le dispositif (II) présentent une diminution de leur défilement sensiblement plus forte que les autres traitements (figures 5 et 6) qui, sauf les témoins du dispositif (l) améliorent cependant déjà naturellement leur forme. Jusqu'à la fin de 1970, dans les deux dispositifs, cette amélioration naturelle ou renforcée par l'élagage persiste mais ralentit ; ensuite, la forme se dégrade à nouveau.

Dans le dispositif (I), cette dégradation semble d'autant plus rapide que le taux d'élagage a été plus fort ; après la saison de végétation 1972, un nouvel élagage inverse le phénomène, favorisant, semble-t-il, une nouvelle tendance naturelle à l'amélioration puisque même le défilement des témoins diminue. Cefte évolution ne dure qu'une saison et après 1973 la forme recommence à se détériorer.

Dans le dispositif (II), l'augmentation du défilement à partir de 1970 affecte surtout le traitement 65 p. 100 puisqu'elle se poursuit jusqu'à la fin de 1973 tout en ralentissant progressivement. Pour les autres traitements, durant la même période, on observe une augmentation faible pendant une saison ou deux, suivie d'une diminution. L'élagage effectué après 1973 provoque une divergence nette entre les traitements 55 p. 100 et 65 p. 100 d'une part qui améliorent à nouveau leur forme, et les autres élagués ou témoins d'autre part dont le défilement se remet à augmenter. 
A la fin de 1975, la situation était la suivante (tabl. 3 et 4) :

Pour le dispositif (I), chaque traitement peut être considéré comme différent des autres au seuil de 5 p. 100 , le défilement des témoins étant tombé à $3,82 \mathrm{~cm} / \mathrm{m}$, celui du traitement 50 p. 100 a $3,68 \mathrm{~cm} / \mathrm{m}$ et celui du traitement $60 \%$ à $3,34 \mathrm{~cm} / \mathrm{m}$. Au seuil 1 p. 100 , seul le traitement 60 p. 100 reste distinct, les deux autres ne pouvant plus être différenciés. La diminution correspondante de défilement en huit saisons de végétation sépare, au seuil de 1 p. 100 , le traitement 60 p. 100 avec, en chiffres arrondis, $0,50 \mathrm{~cm} / \mathrm{m}$, du traitement 50 p. 100 avec $0,14 \mathrm{~cm} / \mathrm{m}$ et des témoins avec $0,10 \mathrm{~cm} / \mathrm{m}$. Les conclusions relatives au défilement calculé entre $1,30 \mathrm{~m}$ et $8 \mathrm{~m}$ sont presque identiques.

Pour le dispositif (II), seul le traitement 65 p. 100 avec un défilement de $3,19 \mathrm{~cm} / \mathrm{m}$ se trouve séparé des autres dont les valeurs sont comprises entre $3,64 \mathrm{~cm} / \mathrm{m}$ et $3,98 \mathrm{~cm} /$ $\mathrm{m}$. La diminution corrélative en sept saisons de végétation fait apparaître une séparation au seuil de 5 p. 100 entre le traitement 65 p. $100(0,86 \mathrm{~cm} / \mathrm{m})$, le traitement 55 p. $100(0,53 \mathrm{~cm} / \mathrm{m})$ et le groupe des trois autres (de $0,17 \mathrm{~cm} / \mathrm{m}$ à $-0,01 \mathrm{~cm} / \mathrm{m})$; au seuil de 1 p. 100 , on assiste au regroupement des deux taux 55 p. 100 et 65 p. 100 et on ne peut même plus dire que la diminution du traitement 55 p. 100 a été plus forte que celle des témoins. Le défilement calculé entre $1,30 \mathrm{~m}$ et $8 \mathrm{~m}$ est un peu plus élevé que celui qui a été calculé entre $1,30 \mathrm{~m}$ et $6 \mathrm{~m}$, mais le classement des traitements reste le même.

L'étude du défilement de 1214 et de son évolution amène un certain nombre de remarques. Comme P. Bonduelle le signale (1971, op. cif.), l'élagage est immédiatement suivi d'une amélioration de la forme des arbres. II faut ajouter que cet effet est net, à condition que l'élagage soit suffisamment marqué : les arbres de nos deux dispositifs réagissent significativement et durablement si les taux adoptés sont supérieurs à 50 p. 100 de la hauteur totale des arbres. Des taux égaux ou inférieurs ne conduisent pas à une amélioration de forme meilleure que celle, naturelle, qu'on observe sur les témoins.

Cette amélioration est d'autant plus forte que le taux d'intensité d'élagage adopté est élevé. C'est incontestablement le taux 65 p. 100 qui conduit à la meilleure forme (diminution du défilement de $0,86 \mathrm{~cm} / \mathrm{m}$ en sept saisons de végétation), devant les taux 60 p. 100 (diminution de $0,50 \mathrm{~cm} / \mathrm{m}$ en huit saisons) et 55 p. 100 (diminution de $0,53 \mathrm{~cm} / \mathrm{m}$ en sept saisons).

Si les élagages ne sont pas renouvelés fréquemment, la tendance à l'amélioration s'estompe progressivement ou même rapidement, parfois même après une seule saison, comme c'est le cas en 1973 pour le dispositif (I).

Les graphiques montrent que la majeure partie et parfois la totalité de l'amélioration de la forme consécutive à une opération d'élagage à taux élevé est acquise dès la première année qui la suit ; à cet égard, les années 1968 et 1973 pour le taux 60 p. 100 du dispositif (I), et 1969 pour les taux 55 p. 100 et 65 p. 100 du dispositif (II) sont démonstratives.

L'élagage devrait être réajusté régulièrement, des passages fréquents permettant de mieux suivre l'évolution des arbres et facilitant chaque opération de taille : ne serait-ce que pour obtenir une amélioration constante de la forme, il faudrait au minimum intervenir tous les deux ans. Les recommandations de P. Bonduelle (1971, op. cit.) sur la conduite de la taille et de l'élagage de I 214 selon lesquelles il 
faut intervenir au début tous les ans dès la fin de la première année de plantation. sont donc tout à fait justifiées. S'il s'écoule trop d'années entre les élagages, en plus de tous les inconvénients qu'il signale (grosses branches à couper, cimes multiples, perte de temps, etc...), le risque est grand de perdre le bénéfice de l'amélioration du défilement, les graphiques semblant montrer que la forme se dégrade d'autant plus vite que son amélioration antérieure a été plus forte.

L'observation de l'évolution naturelle du défilement chez les témoins montre une tendance à sa diminution. Sur cette tendance générale viennent se greffer des accidents annuels, qui font que le défilement, tantôt croît, tantôt décroît, de façon parallèle dans les deux dispositifs. Les traitements à faible taux d'élagage ressemblent de ce point de vue beaucoup aux témoins.

Dans le dispositif (l), les témoins ont une évolution contraire à celle des élagués en 1968, puisqu'ils augmentent leur défilement, alors que l'élagage fait diminuer celui des arbres traités. Les années 1969 et 1970 sont des années favorables à l'amélioration de la forme, puisque les témoins évoluent comme les élagués. Les deux années suivantes semblent avoir une action contraire, les trois traitements augmentant leur défilement avec un effet d'autant plus marqué que le taux d'élagage est fort. La saison 1973 inverse à nouveau la tendance. Par la suite, après les deux années 1974 et 1975 mesurées groupées, tous les arbres du dispositif (I), quel que soit le traitement ainsi que les témoins et les taux 35 p. 100 et 45 p. 100 du dispositif (II) augmentent à nouveau leur défilement ; l'élagage a donc perdu son efficacité au bout d'un an dans le dispositif (I) ef n'a été efficace que pour les taux 55 p. 100 et 65 p. 100 dans le dispositif (II). Malheureusement, comme il n'y a pas eu de mesures da la fin de la saison 1974, on ne peut préciser l'évolution exacte des graphiques pour 1974 et 1975 , les segments de droite reliant 1973 à 1975 n'indiquant qu'une évolution globale sur deux années.

\section{TABLEAU 5}

Dispositif (l). Coefficient de corrélation intratraitement $(r)$ et coefficient de régression (b) de y en $x$ entre le défilement initial ( $x$ ) en 1968 et la diminution totale de ce défilement $(y)$ de 1968 d 1975

(Intratreotment correlation coefficient $(r)$ and regression coefficient $(b)$ from $y$ to $x$ between the initial taper $(x)$ in 1968 (or 1969) and the fofal faper decrease $(y)$ between 1968 (or 1969) and 1975)

\begin{tabular}{|c|c|c|c|c|c|c|c|c|}
\hline & & \multicolumn{2}{|c|}{ Témoins } & 50 & p. 100 & 60 & p. 100 & \\
\hline & $r$ & & 0,52 & & 0,65 & \multicolumn{2}{|r|}{0,71} & \\
\hline & $b$ & & 0,53 & & 0,83 & \multicolumn{2}{|r|}{0,92} & \\
\hline \multicolumn{9}{|c|}{$\begin{array}{l}\text { Dispositif (II). Coefficient de corrélation intratraitement }(r) \text { et coefficient de régression }(b) \text { de y en } \times \text { entre } \\
\text { le défilement inifial }(x) \text { en } 1969 \text { et la diminution fotale de ce défilement }(y) \text { de } 1969 \text { d } 1975\end{array}$} \\
\hline & Témoins & 35 & p. 100 & 45 & p. 100 & 55 & p. 100 & 65 p. 100 \\
\hline$r$ & 0,78 & & 0.76 & & 0.63 & & 0.79 & 0,70 \\
\hline$b$ & 0,90 & & 0,66 & & 0,57 & & 1,00 & 0,99 \\
\hline
\end{tabular}


Si l'on calcule la corrélation entre la valeur initiale du défilement à l'installation du dispositif et la diminution du défilement entre l'installation de 1975, on trouve des coefficients de corrélation significatifs (tabl. 5).

Dans le dispositif (I), le coefficient de corrélation intratraitement, c'est-à-dire calculé sur l'ensemble centré des parcelles unitaires de même traitement, vaut 0,52 dans le cas des témoins et est significatif à 1 p. 1000 ; pour le traitement 50 p. 100 , il atteint 0,65 ef 0,71 pour le traitement 60 p. 100 . Les pentes des droites de régression de la diminution du défilement en fonction de la valeur initiale valent respectivement $0,53,0,83$ et 0,92 .

Dans le dispositif (II), les mêmes coefficients de corrélation calculés pour chaque taux d'élagage sont compris entre 0,63 pour le traitement 45 p. 100 et 0,79 pour le traitement 55 p. 100 ; ils sont tous significatifs à 1 p. 1000 . Les pentes des droites de régression définies comme ci-dessus sont comprises entre 0,57 pour le traitement 45 p. 100 et 1,00 pour le traitement 55 p. 100.

Ces résultats amènent quelques remarques :

- Qu'il y ait élagage ou pas, l'amélioration de la forme est d'autant plus nette que le défilement initial était plus fort puisque tous les coefficients de corrélation calculés sont significatifs et positifs.

- Il semble que pour les traitements dont les résultats sont significativement différents les uns des autres, comme par exemple le taux 60 p. 100 comparé au témoin dans le dispositif (I) ou le taux 65 p. 100 comparé au témoin dans le dispositif (II), il existe des seuils différents de défilement au-dessous desquels il n'y a pas d'amélioration sensible de la forme. Chacun de ces seuils correspondrait à une intensité d'élagage et diminuerait progressivement avec le temps. Lorsque les résultats ne sont pas différents, le seuil est identique, comme dans le cas des témoins et des taux 35 p. 100 et 45 p. 100 du dispositif (II).

- Lorsque le défilement initial est particulièrement faible, et donc voisin de cette limite que nous supposons exister, il peut arriver que, malgré l'élagage, cette caractéristique augmente ; c'est ce qui s'est passé par exemple dans une des parcelles unitaires du dispositif (I) traitée au taux de 50 p. 100, mais dont le défilement initial $3,62 \mathrm{~cm} / \mathrm{m}$ était déjà inférieur au défilement moyen qu'aura ce traitement à la fin de 1975 , soit $3,68 \mathrm{~cm} / \mathrm{m}$. Le traitement 35 p. 100 du dispositif (II) présente un exemple analogue bien que moins marqué.

- En l'absence d'un élagage réajusté régulièrement, les seuils des taux les plus forts, artificiellement abaissés par le traitement, ont tendance à remonter rapidement pour tendre vers les seuils des témoins ou des traitements faibles peu efficaces. L'évolution du traitement 60 p. 100 en 1971 et 1972, puis en 1974 et 1975, ainsi que celle du traitement 65 p. 100 entre la fin de 1970 et celle de 1973 seraient une illustration de ce phénomène.

Le calcul montre qu'il existe un coefficient de corrélation de 0,58 , significatif au seuil'de 5 p. 100 , entre l'intensité du taux d'élagage et la pente de la droite de régression de la diminution du défilement en fonction de sa valeur initiale.

\subsection{Défilement entre $1,30 \mathrm{~m}$ et $8 \mathrm{~m}$.}

Pour le dispositif (I), il a été calculé à partir de 1972 (tableau 3) ; les trois traitements étaient tous différents au seuil de 5 p. 100 parce que l'élagage avait déjà agi 
pendant cinq saisons de végétation. A la fin de 1975, il avait diminué, tout en restant supérieur de $0,30 \mathrm{~cm} / \mathrm{m}$ environ au défilement calculé entre $1,30 \mathrm{~m}$ et $6 \mathrm{~m}$. Seul, le traitement 60 p. 100 a présenté une diminution significativement différente par rapport aux témoins durant ces trois années.

\subsection{Volume.}

\subsection{Volume entre $0 \mathrm{~m}$ et $6 \mathrm{~m}$.}

Dans le dispositif (I), à la fin de 1975, les trois traitements sont tous différents les uns des autres, le volume moyen d'un arbre témoin étant $0,348 \mathrm{~m}^{3}$, celui d'un arbre élagué à 50 p. 100, $0,322 \mathrm{~m}^{3}$ et celui d'un arbre élagué à $60 \%, 0,281 \mathrm{~m}^{3}$. A la même date, dans le cas du dispositif (II), la seule différence observable provient du traitement 65 p. 100 qui, avec un volume d'arbre moyen de $0,268 \mathrm{~m}^{3}$, s'écarte nettement de tous les autres traitements remarquablement groupés entre $0,308 \mathrm{~m}^{3}$ et $0,311 \mathrm{~m}^{3}$.

Les remarques faites à propos des circonférences à $1,30 \mathrm{~m}$ et à $6 \mathrm{~m}$ en ce qui concerne l'influence de l'élagage ef l'influence des années peuvent s'appliquer également à cette caractéristique.

Les figures 7 et 8 montrent l'évolution des volumes dans les deux dispositifs et font preuve d'une parenté de forme évidente avec les figures 1, 2, 3 et 4 relatives aux circonférences.

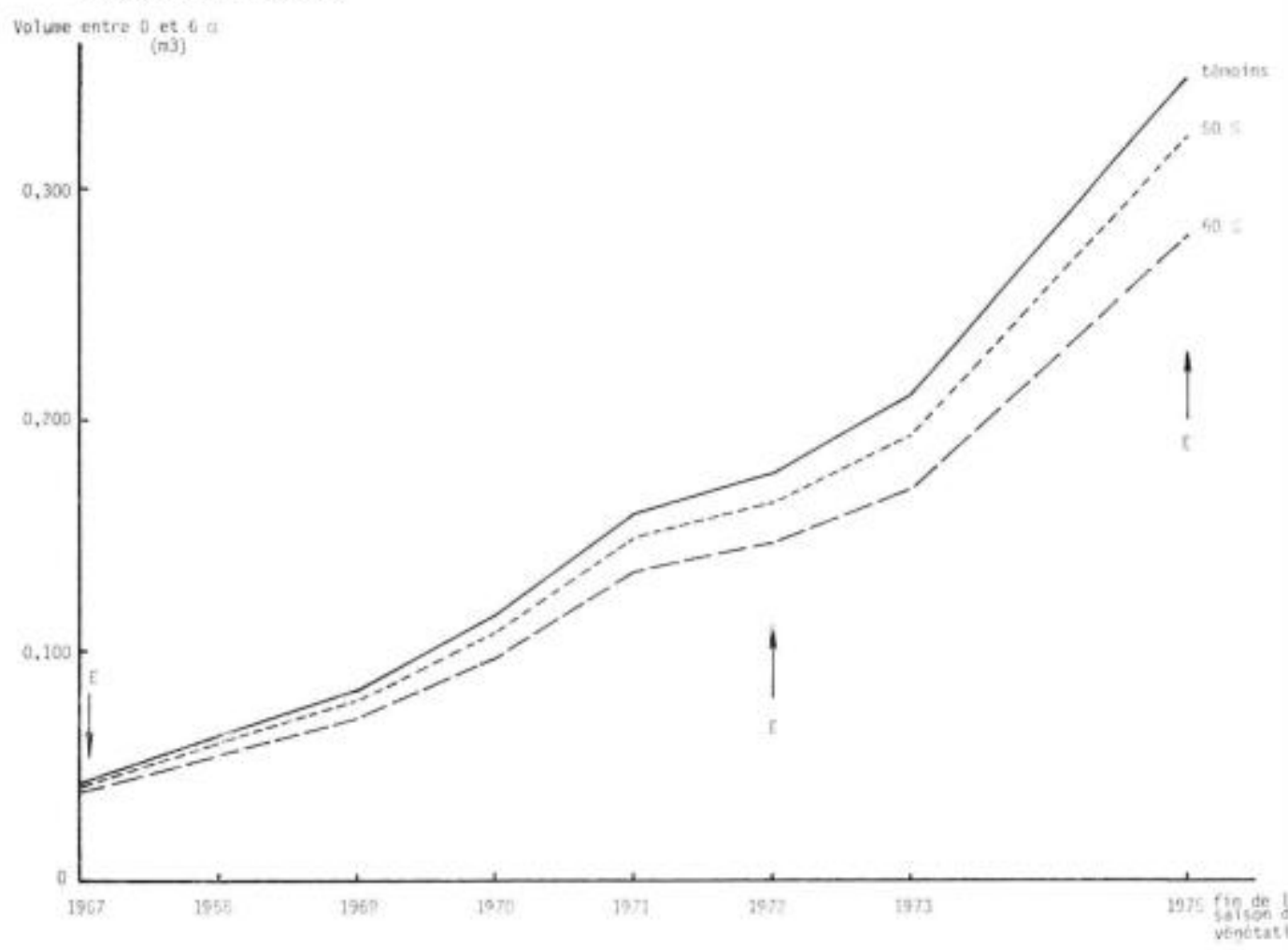

FIG, 7. - Villers-Cotterets. Carré latin. Volume entre 0 ef $6 \mathrm{~m}$.

Villers-Cofferets, latin square. Volume befween 0 and $6 \mathrm{~m}$. 


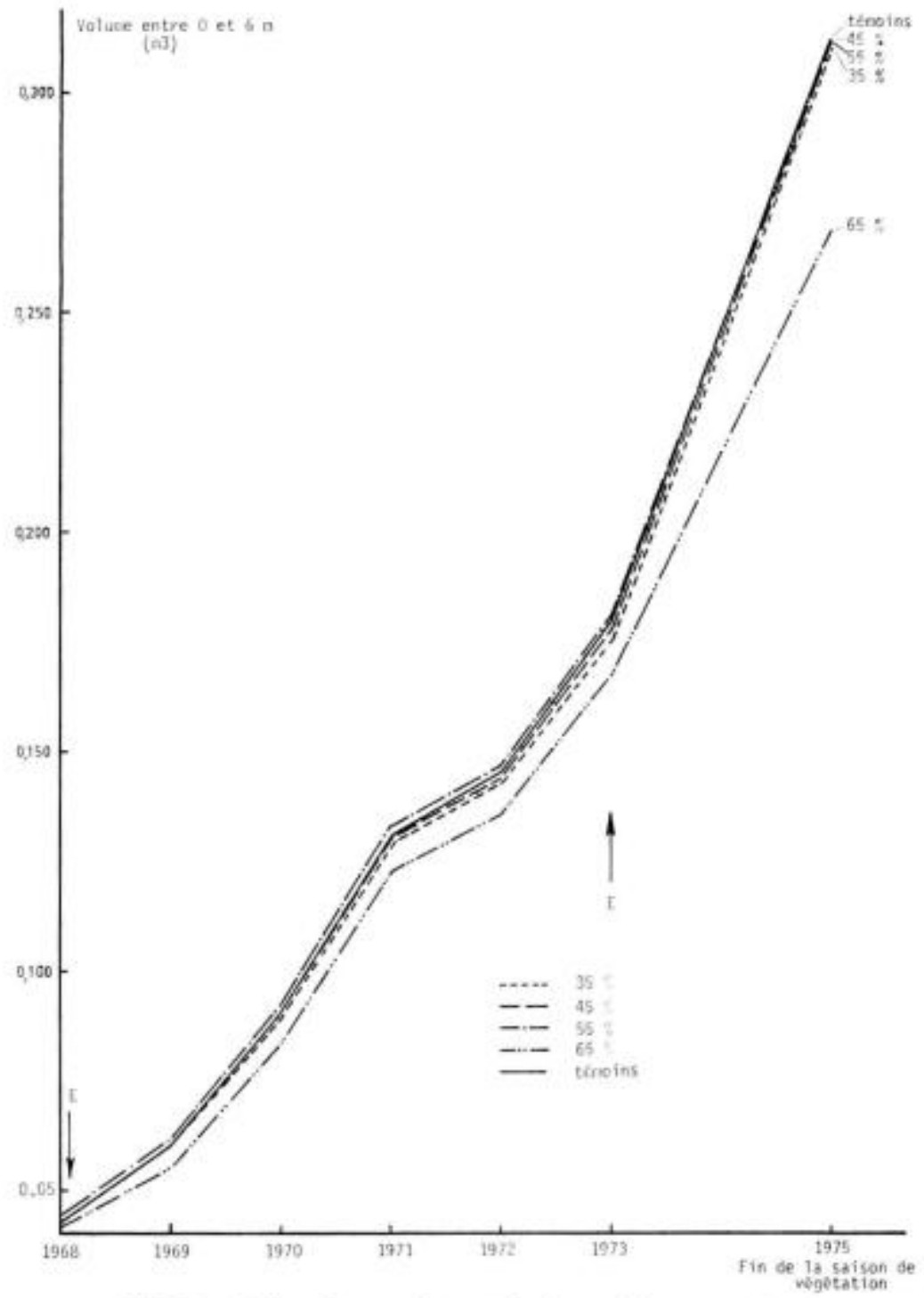

FIG. 8. - Villers-Cotterets. Dispositif en lignes. Volume entre 0 et $6 \mathrm{~m}$. Villers-Cotterets, rows, Volume befween 0 and $6 \mathrm{~m}$.

Pour le dispositif (I), les volumes s'ordonnent en fonction inverse de l'intensité du taux d'élagage dès le début de l'expérience ; les accroissements supérieurs aux témoins des taux 50 p. 100 et 60 p. 100 aux 2/3 de la hauteur initiale ne compensent pas la perte d'accroissement observée à $1,30 \mathrm{~m}$. En huit saisons, les témoins se sont accrus en moyenne, par arbre, de $0,305 \mathrm{~m}^{3}$, les arbres traités à 50 p. 100 de $0,281 \mathrm{~m}^{3}$ (soit 92,1 p. 100 de l'accroissement des témoins) et les arbres traités à 60 p. 100 de $0,242 \mathrm{~m}^{3}$ (soit 79,4 p. 100 de l'accroissement des témoins). Nous n'avons donc pas dans ce cas une évolution aussi favorable que celle qu'a obtenue Bonduelle (1971, op. cit.) 
dans ses peupleraies expérimentales réparties, il est vrai, dans diverses conditions de milieu.

Pour le dispositif (II), le gain de croissance par rapport aux témoins du taux 55 p. 100 aux 2/3 de la hauteur initiale compense largement la perte observée à $1,30 \mathrm{~m}$ pendant les cinq années qui suivent le premier élagage puisque le volume moyen de ce taux est légèrement supérieur à celui des témoins. A la fin de 1975, tous les traitements ont présenté le même accroissement en volume que les témoins (entre $0,265 \mathrm{~m}^{3}$ et $0,268 \mathrm{~m}^{3}$ ) en sept années, seul le traitement $65 \mathrm{p} .100$ se détachant de l'ensemble avec $0,227 \mathrm{~m}^{3}$ ( 84,7 p. 100 de l'accroissement des témoins). Ce dispositif présente une réaction à l'élagage plus avantageuse que le dispositif (I).

\subsection{Volume entre $0 \mathrm{~m}$ et $8 \mathrm{~m}$.}

Dans le cas du dispositif (I), le fait le plus notable est qu'au seuil de 1 p. 100 , il n'est plus possible de distinguer le volume moyen des témoins $\left(0,418 \mathrm{~m}^{3}\right)$ de celui du traitement 50 p. $100\left(0,387 \mathrm{~m}^{3}\right)$. Le traitement 60 p. 100 reste bien séparé avec $0,338 \mathrm{~m}^{3}$, soit 80,9 p. 100 du volume moyen des témoins. Si l'on compare les circonférences à $6 \mathrm{~m}$ et à $8 \mathrm{~m}$ en 1975 , on voit qu'il y a une tendance à ce que les différences entre témoins et traitement 50 p. 100 s'estompent lorsque les mesures se rapprochent de la cime ; cela s'est traduit également dans les valeurs des volumes.

Pour le dispositif (II), tous les traitements sont groupés entre $0,367 \mathrm{~m}^{3}$ et $0,369 \mathrm{~m}^{3}$, sauf encore le traitement 65 p. 100 avec $0,323 \mathrm{~m}^{3}$. La même remarque que pour l'autre dispositif peut être faite : ici le volume du traitement extrême représente 87,8 p. 100 de celvi des témoins ou des autres traitements, ef l'écart s'est réduit en valeur absolue de 2,9 p. 100 par rapport à ce que l'on observe entre $0 \mathrm{~m}$ et $6 \mathrm{~m}$.

\subsection{Diamètre des premières branches vivantes.}

Les branches ont grossi sans que les traitements se différencient, les taux les plus élevés ne provoquant pas un accroissement cccéléré des branches restantes (tabl. 2).

On peut noter que l'évolution du diamètre de ces branches est tout à fait comparable à celle que l'on a observée à propos des circonférences du tronc ou de son volume ; la saison de végétation de 1972, oủ la croissance des grumes a été ralentie, se traduit également par une progression très faible du diamètre des branches.

\section{3. - Nombre de branches coupées ef durée de l'élagage.}

Lors de l'installation du dispositif (I), on a compté le nombre de branches coupées, mesuré leur diamètre à la base et noté le temps nécessaire à l'élagage. Les arbres déjà âgés, rappelons le, avaient été élagués avant la mise en place de l'expérience ef les branches basses des cimes se situaient comme le montre le tableau 6 à $3,5 \mathrm{~m}$ de hauteur. L'élagage a fait remonter théoriquement ce niveau à une valeur égale au produit de la hauteur totale initiale par le taux adopté. En pratique, il faut ajouter à ce produit environ $0,30 \mathrm{~m}$ pour tenir compte du fait que la hauteur réelle d'élagage est presque toujours supérieure à la hauteur calculée, celle-ci ne tombant pas systématiquement juste en-dessous d'une branche ou d'un verticille. Dans ces conditions, I'élagage à 50 p. 100 a fait monter la hauteur de la première branche vivante à environ $5,15 \mathrm{~m}$ et l'élagage à 60 p. 100 à environ $6,10 \mathrm{~m}$. 
TABLEAU 6

Dispositif (1). Hauteur d'élagage, nombre de branches coupées ef temps d'élagage à l'installation (Plot (I). Pruning height, number of cut branches and pruning duration at the experiment installation)

\begin{tabular}{|c|c|c|c|c|c|c|}
\hline \multirow{2}{*}{$\begin{array}{c}\text { Traite- } \\
\text { ment } \\
\text { Treat- } \\
\text { ment }\end{array}$} & \multicolumn{3}{|c|}{$\begin{array}{l}\text { Hauteur moyenne en m des } \\
\text { premières branches basses } \\
\text { de la cime avant la saison } \\
\text { de végétotion de } 1968 \\
\text { (meon height in metre } \\
\text { of the first lower branches of the } \\
\text { crown before the vegetation } \\
\text { season of 1968) }\end{array}$} & \multirow{2}{*}{$\begin{array}{c}\text { Nombre total } \\
\text { de branches } \\
\text { coupées } \\
\text { (total number } \\
\text { of cut branches) }\end{array}$} & \multirow{2}{*}{$\begin{array}{l}\text { Nombre de branches } \\
\text { coupćes de diamètre } \\
\text { compris entre } \\
2,5 \mathrm{~mm} \text { et } 42,5 \mathrm{~mm} \\
\text { (number of cut bran- } \\
\text { ches with a diameter bet- } \\
\text { ween } 2,5 \text { and } 42,5 \mathrm{~mm} \text { ) }\end{array}$} & \multirow{2}{*}{$\begin{array}{l}\text { Temps moyen } \\
\text { d'élagage } \\
\text { scie ou élagoir } \\
\text { (mean duration of } \\
\text { pruning, pole } \\
\text { pruning saw or } \\
\text { tree trimmer) }\end{array}$} \\
\hline & $\begin{array}{c}\text { avant } \\
\text { élagage } \\
\text { before } \\
\text { (pruning) }\end{array}$ & $\begin{array}{l}\text { theorique } \\
\text { après } \\
\text { élagage } \\
\text { (theore- } \\
\text { tical } \\
\text { height } \\
\text { after } \\
\text { pruning) }\end{array}$ & $\begin{array}{l}\text { réelle } \\
\text { après } \\
\text { élagage } \\
\text { (actual } \\
\text { height } \\
\text { after } \\
\text { pruning) }\end{array}$ & & & \\
\hline $\begin{array}{l}\text { Témoins } \\
\text { (Control } \\
\text { trees) ... }\end{array}$ & 3,47 & - & 3,47 & - & - & - \\
\hline 50 p. 100. & 3,51 & 4,87 & 5,17 & 21 & 17 & $1 \mathrm{mn} 59 \mathrm{~s}$ \\
\hline 60 p. 100. & 3,50 & 5,78 & 6,08 & 29 & 23 & $2 \mathrm{mn} 27 \mathrm{~s}$ \\
\hline
\end{tabular}

$\mathrm{mn}=$ minute, $\mathbf{s}=$ seconde.

La figure 9 montre la répartition des branches coupées de diamètre supérieur à $2,5 \mathrm{~mm}$ et inférieur ou égal à $42,5 \mathrm{~mm}$. Un peu plus de $90 \mathrm{p}$. 100 des branches enlevées avaient un diamètre inférieur ou égal à $22,5 \mathrm{~mm}$, pour le traitement 50 p. 100 , et $25 \mathrm{~mm}$ pour le traitement 60 p. 100 . Le nombre total de branches coupées est évidemment un peu supérieur au nombre des branches coupées comprises entre certaines dimensions ; la différence est le fait de très petites branches inférieures à $2,5 \mathrm{~mm}$ de diamètre. N'ont pas été prises en compte ici les branches coupées au-dessus de la limite d'élagage pour former la cime ; cette opération nécessaire a d'ailleurs pris beaucoup de temps pour certains arbres puisqu'elle a commencé 6 ans après l'installation des plants, donc beaucoup trop tardivement (fig. 10 et 11).

Le fableau 6 montre que, pour le traitement 50 p. 100, on a coupé par arbre en moyenne dix-sept branches de diamètre supérieur à $2,5 \mathrm{~mm}$ pour passer de $3,50 \mathrm{~m}$ à $5,15 \mathrm{~m}$ de hauteur de fût sans branches, ce qui représente environ dix branches par mètre d'élagage. Pour le traitement 60 p. 100 , il a fallu couper vingt-trois branches pour passer de $3,50 \mathrm{~m}$ d̀ $6,10 \mathrm{~m}$, soit environ neuf branches par mètre.

Le femps moyen d'élagage par arbre a été de $1 \mathrm{mn} 59 \mathrm{~s}$ pour le traitement $50 \mathrm{p}$. 100 et $2 \mathrm{mn} 27 \mathrm{~s}$ pour le traitement $60 \mathrm{p}$. 100, compte non tenu du temps de déplacement d'un arbre à l'autre, que l'on peut estimer à environ 10 p. 100 du temps d'élagage proprement dit. 

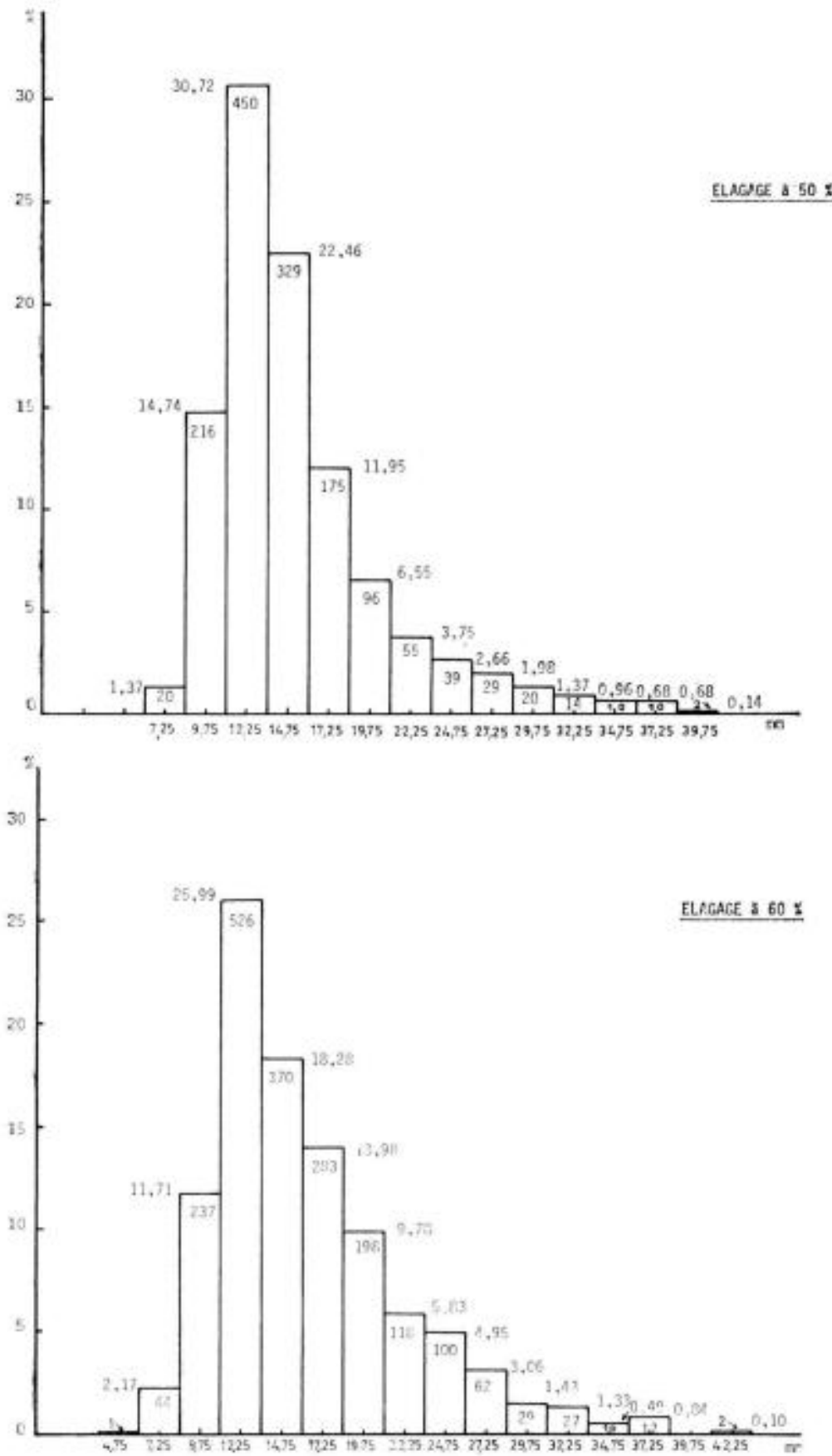

FIG. 9. - Villers-Cotferets. Carré latin : Répartition des branches coupées en classes de diamètre à l'insfallation du dispositif (printemps 1968).

Villers-Cotterets, latin square. Histogram of cut branches in diameter classes at the experiment installation (spring 1968). 
Par la suite, ce premier dispositif fut réélagué à l'automne 1972, puis au printemps 1976. Les mesures faites lors de ce dernier traitement sont regroupées dans le tableau 7.

Dans le traitement 50 p. 100 , pour passer d'une hauteur d'élagage de $8,10 \mathrm{~m}$ environ à $10,35 \mathrm{~m}$, il a fallu couper huit branches par arbre, ce qui a pris un temps de $16 \mathrm{mn}$ pour un opérateur.

Dans le cas du traitement 60 p. 100 , pour passer de $9,40 \mathrm{~m}$ à $12,10 \mathrm{~m}$, on a coupé environ onze branches par arbre et cela a pris en moyenne $27 \mathrm{mn}$ et $22 \mathrm{~s}$. Les arbres ont tous été élagués à partir d'échelles, soit à la scie égoine, soit à l'aide d'une tronçonneuse légère ; les temps indiqués comprennent les temps de toutes les opérations

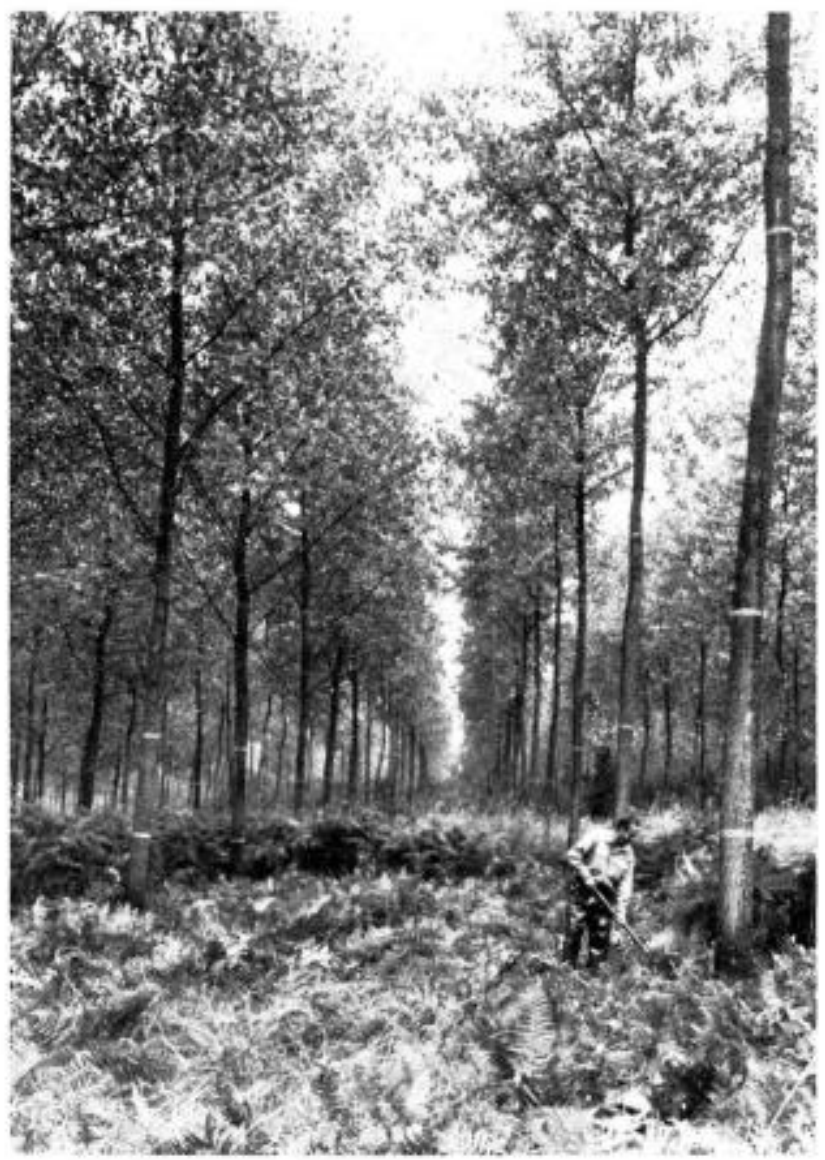

FIG. 10. - Villers-Cofferets. Carré latin :

d̀ droite : ligne de peupliers élagués à 60 p. 100.

à gouche : ligne de peupliers têmoins

(état en août 1975).

Villers-Cotterets, latin square (August 1975).

on the right : row of pruned poplars, at 50 p. 100 pruning intensity.

on the left : row of control poplars, dry pruned only 


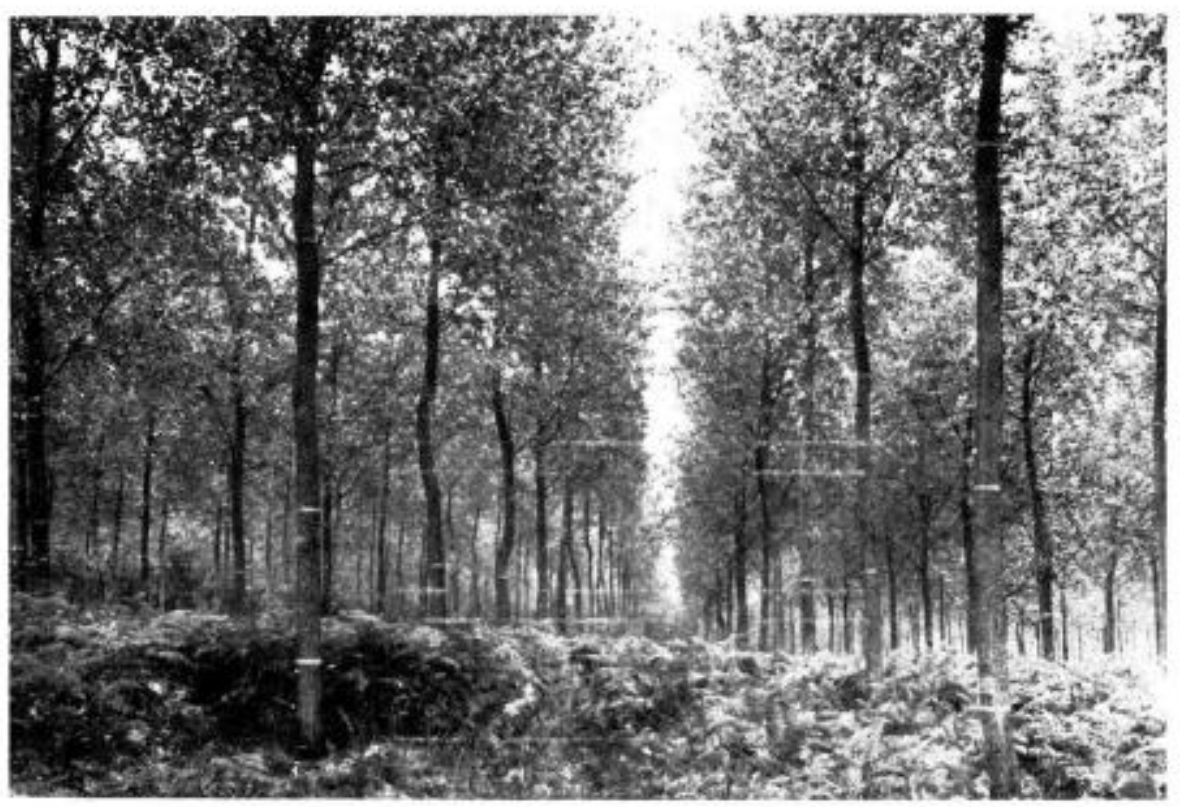

FIG. 11. - Villers-Colterets. Carré latin :

à droite : ligne de peupliers élagués d 50 p. 100.

à gauche : ligne de peupliers témoins

(étât en aoôt 1975).

Villers-Cotterets, latin square (August 1975).

on the right : row of pruned poplors, of 60 p. 100 pruning intensity.

on the left : row of control, dry pruned only.

Remarque concernant les figures 10 et 11 .

Certains arbres présentent une forme très défectueuse du fait que l'élagage ef la formation des cimes ont commencé trop tardivement.

Remark about plates 2 and 3

Some trees show a very defective form because pruning and crown formation have begun too late.

annexes : déplacements et installation des échelles, mise en service de la tronçonneuse au niveau de l'élagage, etc... L'élagage à grande hauteur dans ces conditions prend donc un temps considérable.

\section{4. - Influence de l'élagage sur quelques propriétés du bois.}

En octobre 1969, dans le dispositif (1), on a sondé à la tarière de Pressler de $5 \mathrm{~mm}$ de diamètre, quinze arbres témoins et quinze arbres élagués à 60 p. 100 . Chaque arbre a fourni deux carottes diamétrales orientées nord-ovest, sud-est évitant autant que possible le bois de tension, l'une prise à 1,30 m et l'autre aux $2 / 3$ de la hauteur totale initiale, soit environ $6,50 \mathrm{~m}$. Ces échantillons ont été radiographiés et leurs 


\section{TABLEAU 7}

Dispositif (1). Hauteur d'élagoge nombre de branches coupées et temps d'élagage ou printemps 1976

(Plot (I). Pruning height, number of cut branches and pruning duration in spring 1976)

\begin{tabular}{|c|c|c|c|c|c|}
\hline \multirow{2}{*}{$\begin{array}{l}\text { Traitement } \\
\text { Treatment }\end{array}$} & \multicolumn{3}{|c|}{ 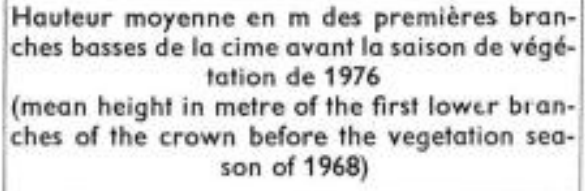 } & \multirow{2}{*}{$\begin{array}{c}\text { Nombre total } \\
\text { de branches coupées } \\
\text { (total number of cut } \\
\text { branches) }\end{array}$} & \multirow{2}{*}{$\begin{array}{l}\text { Temps moyen d'éla- } \\
\text { gage scie égoïne ou } \\
\text { tronçonneuse } \\
\text { (mean duration of } \\
\text { pruning, hand saw or } \\
\text { chain saw }\end{array}$} \\
\hline & $\begin{array}{l}\text { avant } \\
\text { élagage } \\
\text { (before } \\
\text { pruning) }\end{array}$ & $\begin{array}{c}\text { théorique } \\
\text { après élagoge } \\
\text { (theoretical } \\
\text { height after } \\
\text { pruning) }\end{array}$ & $\begin{array}{l}\text { réelle après } \\
\text { élagage } \\
\text { (actual height } \\
\text { after pruning) }\end{array}$ & & \\
\hline 50 p. $100 \quad \ldots$ & 8,08 & 9,85 & 10,33 & 8 & $16 \mathrm{mn}$ \\
\hline 60 p. $100 \ldots$ & 9,39 & 11,62 & 12,10 & 11 & $27 \mathrm{mn} 22 \mathrm{~s}$ \\
\hline
\end{tabular}

images analysées au microdensitomètre selon la méthode classique de notre laboratoire. Le tableau 8 regroupe les valeurs de quatre données mesurées dans le bois formé en 1966 et 1967, années précédant l'élagage, et celui de 1968 et 1969.

\subsection{Etat initial,}

Dans le bois de 1966, il n'existe à 1,30 m aucune différence entre les valeurs des largeurs de cernes, des densités minimales $d$, des densités maximales $D$ et de l'hétérogénéité $D$-d. Les valeurs correspondantes à $6 \mathrm{~m}$ pour cette année n'ont pu être mesurées, le cerne en question étant à ce niveau proche de la mcelle et les carottes plus ou moins excentrées.

Pour le bois de 1967, il n'existe pour les deux niveaux aucune différence, sauf pour la largeur de cerne à $1,30 \mathrm{~m}$, le futur traitement à 60 p. 100 présentant une croissance sur le rayon de $8,0 \mathrm{~mm}$ contre $9,3 \mathrm{~mm}$ pour le futur traitement témoin.

\subsection{Evolution observée une année après l'élagage.}

Au cours de 1968, première saison de végétation après l'élagage, on observe une certaine évolution de ces caractères :

\subsection{Largeur de cerne.}

A $1,30 \mathrm{~m}$, la dépression déjà observée en 1967 et probablement renforcée par l'élagage, se manifeste nettement puisque les témoins s'accroissent de $11 \mathrm{~mm}$ sur le rayon contre $7,4 \mathrm{~mm}$ pour les arbres traités à 60 p. 100.

A $6,5 \mathrm{~m}$, le phénomène est inversé ; ce sont les arbres élagués qui croissent le plus vite avec $11,6 \mathrm{~mm}$ contre $9,4 \mathrm{~mm}$ pour les témoins.

L'échantillonnage reflète donc l'aspect des figures 1 et 3 tracées pour l'en- 


\section{TABLEAU 8}

Influence de l'élagage sur quelques propriètés du bois Influence of the pruning on some wood properties (microdensitometry)

- a $1,30 \mathrm{~m}$ (at $1,30 \mathrm{~m}$ level)

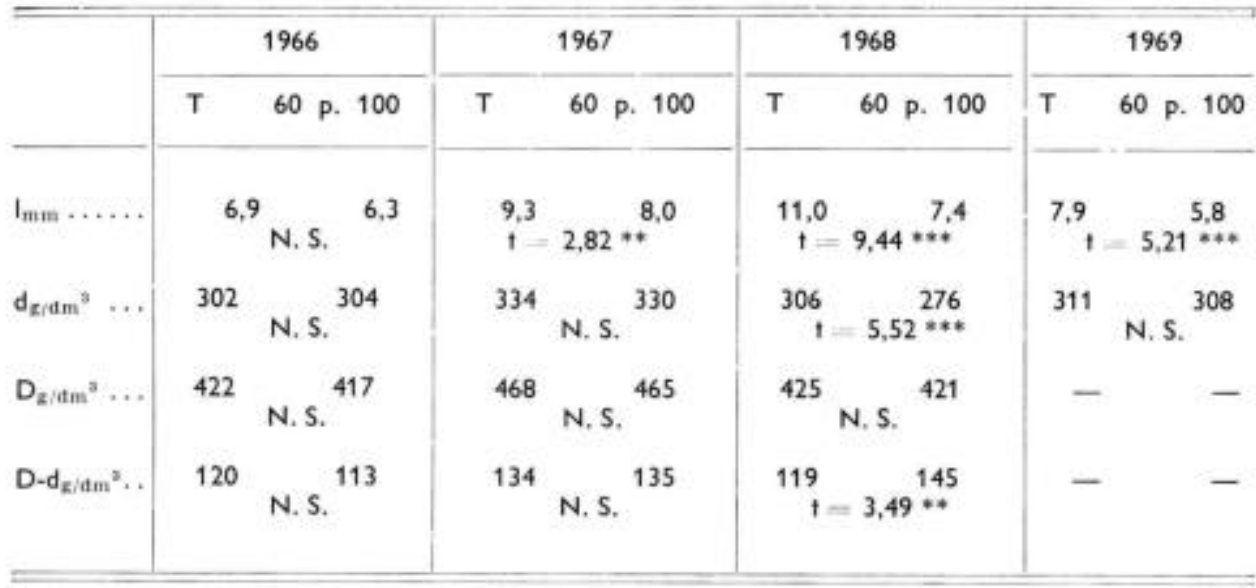

- à $6,50 \mathrm{~m}$ (at $6,50 \mathrm{~m}$ level)

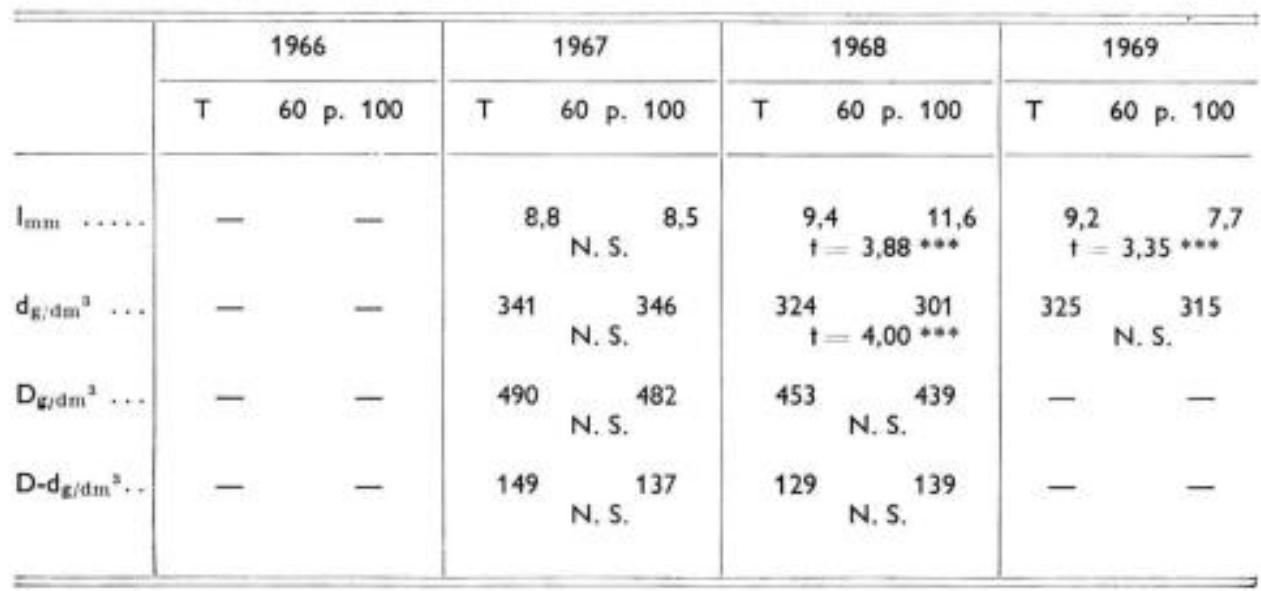

$T$ - control tree.

60 p. $100=60$ p. 100 total height pruned tree,

Imam - largeur de cernes en $\mathrm{mm}$ (ring width in millimetres.)

$\mathrm{dg} / \mathrm{dma}=$ densité minimum en $\mathrm{g} / \mathrm{dm}^{3}$ (minimal annual density in gram per cubic decimetre.)

$\mathrm{D}_{\mathrm{g} / \mathrm{dm}, \mathrm{m}}=$ densite maximum en $\mathrm{g} / \mathrm{dm}^{3}$ (maximal annual density in gram per cubic decimetre.)

$t=1-$ test, NS - not significant,

$+=$ significant at 5 p. 100 level.

* - significant at 1 p. 100

$* *$ = significant at 1 p. 1000 level.

D-da/dm $\mathrm{m}^{3}=$ mesure de l'hétérogénété en $\mathrm{g} / \mathrm{dm}^{3}$ (estimation of annual wood heterogeneify in gram per cubic decimetre.) 
semble de la population mesurée où l'on voit en 1968 un ralentissement de la croissance d̀ $1,30 \mathrm{~m}$ et une accélération à $6,50 \mathrm{~m}$ au voisinage de la base de la cime.

\subsection{Densité minimale.}

Par rapport à 1967, cette caractéristique diminue à la fois chez les témoins et les arbres élagués, mais elle diminue plus pour ces derniers, cussi bien à $1,30 \mathrm{~m}$ qu'à $6,50 \mathrm{~m}$, ce qui fait apparaître des différences significatives.

\subsection{Densité maximale.}

Sa décroissance par rapport à 1967 est à peu près identique pour les deux traitements et il n'apparait pas entre eux de différences en 1968, pour aucun des deux niveaux.

\subsection{Hétérogénéité.}

A 1,30 m, elle diminue chez les témoins par rapport à 1967 et elle augmente chez les élagués si bien qu'il apparait une différence significative. A $6,50 \mathrm{~m}$, la diminution s'observe encore chez les témoins, mais les élagués restent da peu près constants ; on n'observe pas de différence entre les deux traitements.

\subsection{Evolution observée deux années après l'élagage.}

Pour l'année 1969, seules la largeur de cerne et la densité minimale ont pu être mesurées. En effet, les sondages ont eu lieu en octobre 1969 et l'opération a légèrement détérioré le bois correspondant à la fin de la saison de végétation, ce qui a empêché la mesure de la densité maximale.

\subsection{Largeur de cerne.}

A $1,30 \mathrm{~m}$, elle reste inférieure chez les élagués à ce qu'elle est chez les témoins.

A $6,50 \mathrm{~m}$, l'accélération de croissance observée chez les élagués ne se maintient pas ; on assiste chez eux au contraire à un ralentissement significatif ef les témoins les devancent (la figure 3 montre que sur l'échantillonnage total, ce ralentissement ne se manifeste pas aussi fortement puisque les témoins ne dépassent les élagués qu'en 1970).

\subsection{Densité minimale.}

On n'observe plus de différence entre traitements, ni à $1,30 \mathrm{~m}$, ni à 6,50 m.

Ces premières observations, bien que très limitées, sur l'influence de l'élagage sur les propriétés du bois du peuplier 1214 montrent cependant que ce feuillu ne réagit pas de la même façon que le douglas ou le grandis à ce traitement $(\mathrm{H}$. Polge, R. Keller, F. Thiercelin, 1973) en ce qui concerne les densités minimales et maximales. En effet, ces deux résineux, mais surtout le grandis, forment, après élagage de branches vivantes, du bois à plus forte densité minimale et maximale, ce phénomène pouvant persister plusieurs années après le traitement ; or chez ce peuplier, la densité maximale (mesurée il est vrai sur une seule année, juste après l'élagage) n'est pas affectée et la densité minimale des élagués diminue par rapport à celle des témoins la première année suivant l'opération, puis reprend des valeurs comparables pour les deux traitements dès la deuxième saison de végétation. 


\section{4. - Discussion et conclusion}

\section{1. - Amélioration de la forme}

Comme cela a été observé déjà de nombreuses fois, l'élagage du peuplier 1214 provcque une amélioration de la forme des fûts par diminution du défilement (Pourtet, 1961). Cette amélioration est cependant assez fugace et, pour la rendre permanente, il faut poursuivre régulièrement l'élagage et adopter un taux assez fort.

\section{2. - Croissance en hauteur}

La croissance en hauteur dans nos dispositifs n'a pas été freinée par l'élagage et on a même pu observer qu'une tendance se manifestait selon laquelle les arbres élagués fortement auraient une croissance en hauteur un peu plus forte que les témoins : sans que l'on puisse dire toujours que l'élagage provoque un accroissement en hauteur ( $P$. Bonduelle, op. cit.), nos mesures vont dans le sens des observations de cet auteur dans l'un des deux dispositifs et pour certains taux élevés.

\section{3. - Accroissement en circonférence}

L'élagage provoque un ralentissement de l'accroissement en circonférence dans la partie inférieure du tronc. Mais, au voisinage de la cime vivante, ou bien ce phénomène s'estompe, ou même il s'inverse, et dans certains cas, comme les taux 45 p. 100 ou 55 p. 100 du dispositif (II), les arbres élagués égalent ou dépassent les témoins. Lorsque le taux est très élevé, comme les taux 60 p. 100 du dispositif (I) et 65 p. 100 du dispositif (II), l'effet dépressif de l'élagage se manifeste à tous les niveaux.

\section{4. - Accroissement en volume}

La production en volume est faiblement affectée par l'élagage : les arbres élagués à 50 p. 100 du dispositif (l) ont eu un accroissement en volume égal à 92 p. 100 de celui des témoins dans les six premiers mètres du fût, ou pas du tout affectée, comme dans le cas du dispositif (II) où les taux 35 p. 100, 45 p. 100 et 55 p. 100 ont eu le même accroissement que les témoins. Ce n'est que lorsque le taux atteint ou dépasse $60 \mathrm{p}$. 100 que la perte de production devient sensible. Nous retrouvons là les conclusions de Frison (1975) ou de Bonduelle (1971, op. cit.) selon qui « un élagage normal du peuplier 1214 ne gêne en rien l'accroissement en volume des fûts » et apportons peutêtre une vérification de l'hypothèse de Deveaux (1969) qui, par des considérations sur la transpiration foliaire des arbres et ses modifications dues à l'élagage, écrivait « il n'est pas exclu que dans bien des cas cette opération, indépendamment du gain de valeur au mètre cube, soit susceptible d'accélérer l'accroissement en volume de l'arbre au lieu de le réduire, si bien entendu elle reste tout de même dans des limites raisonnables $\gg$.

Nous n'avons pas, à strictement parler, observé des augmentations d'accroissement en volume, mais des augmentations d'accroissement en circonférence à certains niveaux compensant des diminutions à d'autres et, par conséquent, des accroissements en volume identiques, que les arbres soient élagués ou non. En outre, pour une lon- 
gueur égale de fôt, ce volume d'arbre élagué est plus intéressant au débit que le même volume d'un arbre non élagué puisque le défilement est plus faible. Indépendamment de l'absence de ncuds, de la meilleure rectitude du fil du bois, on gagnera aussi sur le rendement au déroulage $(H$. Polge, 1969).

\section{5. - Qualité du bois}

Pour que l'étude de l'élagage soit complèłe, il faudrait prendre en considération, en plus de ces caractères de croissance, des caractères de qualité du bois comme nous avons tenté de le faire. On a vu que l'élagage pouvait avoir une influence sur la densité minimale ou l'hétérogénéité du bois ; nos données ne nous permettent pas de dire si cette action est durable. Après une dizaine d'années de végétation, de nouveaux sondages à la tarière pour continuer les études microdensitométriques devraient permettre d'y voir un peu plus clair. II semble toutefois que la réaction de ce feuillu en ce domaine soit différente de celle des résineux qui ont déjà pu être étudiés de ce point de vue.

D'autres observations importantes devraient être faites. II a été montré que chez des peupliers « Regenerata», « Robusta», « Oxford», « Rochester», « Wettstein 264 » (Sachsse, 1965) et à nouveau « Robusta » (Sacre, 1975), la proportion de faux bois de cœur et de bois de tension (en section et en hauteur dans le fût) chez les arbres élagués était plus importante que chez les arbres non élagués. Les résultats donnés par ce dernier auteur sont toutefois à considérer comme des éléments provisoires puisque, comme il l'indique, les arbres élagués et les non élagués étaient plantés à des écartements différents dans des sols différents.

II se pourrait aussi que cette formation en plus grande quantité du bois de tension chez les arbres élagués contraigne à limiter la pratique de l'élagage aux n premières années de la vie des arbres, n devant être déterminé en fonction des clones et des conditions de milieu (van Buggenhoudt, 1954 dans Sachsse, 1965, op. cit.). Dans ces conditions, on aurait intérêt à élaguer tôt et régulièrement et à adopter des taux d'élagage assez élevés, mais restant compatibles avec une bonne croissance pour atteindre des hauteurs suffisantes nettes de nœuds, tout en cantonnant ces défauts dans la partie la plus interne des troncs. Cette pratique permettrait aussi de ne couper que des branches d'assez faible diamètre, ce qui ne peut qu'être favorable à tous points de vue, d'autant plus que, selon van Buggenhoudt, la proportion de bois de tension serait plus forte lorsque le diamètre des branches coupées est plus grand.

Dans nos dispositifs de Villers-Cotterets, où sur la même station se trouvent les les différents traitements, la vérification de ces résultats sur 1214 pourrait être entreprise à partir de sondages à la tarière à différents niveaux des troncs.

\section{6. - Taux à adopter}

La gradation des taux d'élagage adoptée dans nos deux dispositifs permet de préciser ce que peut être un «élagage normal » et ses « limites raisonnables». On a vu qu'il y avait quelques différences de comportement dans ces deux dispositifs mais dans le premier, un taux de 50 p. 100 n'a provoqué qu'une faible perte de production de l'accroissement en volume par rapport aux témoins, environ 8 p. 100 en 8 ans, 
et dans le second, il n'y a eu aucune perte de production même dans le cas du traitement à 55 p. 100 . Seuls les taux de 60 p. 100 et 65 p. 100 provoquent des pertes importantes sur l'accroissement en volume ; en contrepartie, leur amélioration de forme par diminution sensible du défilement est la plus satisfaisante. La diminution du défilement entre $1,30 \mathrm{~m}$ et $6 \mathrm{~m}$ dans le dispositif (I) au bout de huit années pour le taux 50 p. 100 n'est pas marquée $(0,144 \mathrm{~cm} / \mathrm{m})$ et pas statistiquement différente de celle des témoins $(0,098 \mathrm{~cm} / \mathrm{m})$; elle est nette en ce qui concerne le taux 60 p. $100(0,496 \mathrm{~cm} /$ $\mathrm{m}$ ). Dans le dispositif (II) au bout de 7 années, cette diminution apparaît avec le taux 55 p. $100(0,530 \mathrm{~cm} / \mathrm{m})$. Si l'on ne veut pas de perte de production, ou tout au moins une perte relativement faible, tout en continuant d'améliorer la forme des fûts, il faut donc adopter un taux d'élagage de 50 d̀ 55 p. 100 environ de la hauteur totale des arbres. Ceci permet de modifier, pour notre cas particulier, le schéma de Pourtet (1961, op. cit.) préconisant, en règle générale, des élagages sur le tiers de la hauteur totale des arbres entre 5 et 10 ans, sur la moitié de 10 à 15 ans, et éventuellement sur les 2/3 au-delà.

Dans des conditions analogues de sol et de densité de plantation, des élagages sensiblement plus intenses peuvent donc être envisagés sur I 214 puisque des taux élevés, appliqués sur des arbres âgés de 7 à 8 ans à l'installation de l'expérience ef renouvelés ont donné des résultats satisfaisants sur des arbres âgés aujourd'hui d'une quinzaine d'années.

Pour des raisons diverses, l'installation de nos dispositifs fut faite sur des peuplements déjà trop âgés et, par la suite, la fréquence des réajustements des taux d'élagage fut trop faible ; il en est résulté des difficultés pour obtenir des arbres de forme satisfaisante, I 214 ayant tendance à fourcher et à être sinueux. Mises à part ces questions de fourches et de sinuosité, tous les résultats présentés restent intéressants à la fois d'un point de vue théorique et pratique.

Les recommandations de Bonduelle (1971, op. cit.) relatives à la pratique de la taille et de l'élagage du peuplier I 214 doivent être bien suivies, sachant qu'on peut dans des conditions comparables à celles de nos dispositifs, se permettre de couper toutes les branches basses jusqu'à une hauteur égale à 50-55 p. 100 de la hauteur totale des arbres. Si l'on adopte des faux plus faibles, lors des réajustements successifs, on est amené à couper des branches dont certaines sont sèches et l'opération n'aura qu'une répercussion limitée sur l'évolution du défilement (lors du deuxième élagage du dispositif (II) en 1974, soit 5 ans après son installation, on a coupé 77 p. 100 de branches mortes dans le cas du taux 35 p. 100,42 p. 100 pour le taux 45 p. 100 , 30 p. 100 pour les taux 55 p. 100 , et 17 p. 100 pour le taux 65 p. 100) puisque seul l'enlèvement de branches vivantes a une action contrôlée sur la physiologie des arbres. Le dépérissement naturel provoque évidemment également une réaction, mais non contrôlée et quand on intervient à intervalles trop éloignés, outre le fait qu'on ne peut convenablement orienter harmonieusement le développement des arbres, cela risque de donner l'impression que l'intervention a peu d'effet sur l'amélioration de la forme puisque celle-ci a déjà commencé d'elle-même.

Enfin, il faut bien insister sur le fait que les chiffres que nous avançons ne peuvent être de quelque utilité que dans des conditions de plantation ef de milieu comparables à celles qui existent là où sont les deux dispositifs éfudiés, et comme le dit Deveaux (1969, op. cit.), il est probable que l'intensité de l'élagage ne doit pas être la même dans toutes les conditions de milieu et de traitement. 
Une autre limite raisonnable est imposée par la hauteur jusqu'à laquelle on peut élaguer sans passer à cette opération un temps prohibitif. Pour élaguer jusqu'à $6 \mathrm{~m}$, il n'est besoin que de 2 à $3 \mathrm{mn}$ par arbre. On a vu que si on voulait monter l'élagage de $8 \mathrm{~m}$ à $10 \mathrm{~m}$, ce temps atteignait environ $16 \mathrm{mn}$ et que si on voulait passer de $9,5 \mathrm{~m}$ $12 \mathrm{~m}$, il fallait près d'une demi-heure. A moins peut-être d'employer d'autres matériels. on peut provisoirement considérer qu'avec des moyens tels que ceux que nous avons utilisés, on ne peut économiquement dépasser une hauteur de $10 \mathrm{~m}$ d'élagage. Une étude économique complète sur la question permettrait sans doute de mieux préciser encore dans diverses hypothèses la limite raisonnable à ne pas dépasser.

Reçu pour publication en juillet 1978.

\title{
Remerciements
}

Cette étude de l'élagage du peuplier I 214 a été possible grâce à Monsieur M. Vadet, Chef du Centre de Gestion de l'Office National des Forêts de Villers-Cotterets, qui a grandement facilité l'implantation des dispositifs en forêt domaniale de Retz, ainsi que toutes les opérations ultérieures.

Qu'il soit remercié pour son aide précieuse ef l'intérêt qu'il porte à ces expérimentations.

\section{Summary}

\author{
First results of a pruning experiment on 1-214 poplar \\ (Populus $\times$ euramericana (DODL) GUINIER cr. " $1-214 »$ )
}

7-year old poplars were green pruned with varying intensities. The lower branches were cut up to a height proportional to the total height of the tree. Rates of 35, 45, 50, 55, 60 and 65 p. 100 of this height were selected. Seven of eight years later, the main results are as follows :

- Pruning improves the form of trunks by taper reducing taperapering of the stem : the higher the pruning intensity, the more pronounced this effect.

- Height growth is not slowed down by pruning.

- Pruning causes a decrease in girth growth in the lower parts of the trunks. Close to the base of the crown this decrease is less marked. Intensities of 60 and 65 p. 100 only have a depressive effect on girth growth throughout the length of the frunk.

- Volume growth is slightly reduced by pruning ; intensities of 60 and 65 p. 100 only produce an obvious yield loss. However, this loss is partly compensated at sawing by the better from of the boles which are more cylindrical.

- For environmental conditions comparable to those of the present experiment, it is recommended to prune up to a total tree height of 50 to 55 p. 100 . Pruning must begin at an early stage and regulariy control the evolution of the trees. In the case of \& 1214 " pruning must be complemented by the removal of multiple leaders and by cutting away branches that are too large in the crown, from the first years following planting.

- Special attention should be directed in the coming years to the influence of pruning intensity on the formation of tension wood and possible development of calcured heartwood.

\section{Zusammenfassung}

\author{
Erste Ergebnisse eines Ästungsversuches der Pappelsorte I 214 \\ (Populus x euramericana (DODE) Guinier cv. « I $214 »)$
}

Siebenjährige Pappeln 1214 wurden nach verschiedenen Prozentsätzen geästet. Die niedrigen Aste wurden bis zu einer Höhe entfernt, die der Gesamthöhe der Bäume entsprach. So wurden Prozentsätze von $35,45,50,55,60$ und 65 p. 100 der Gesamthöhe gewählt. 
Nach sieben bzw. acht Wachstumszeiten ergaben sich folgende Hauptergebnisse :

- Die Ástung führt zu einer Minderung der Durchmesserabnahme, was eine Verbesserung der Form der Stämme bedeutet. Je höher der Prozentsatz der Ástung, desto entschiedener die Wirkung.

- Der Höhenzuwachs wird keinesfalls durch die Ästung verlangsamt.

- Die Ástung bewirkt eine Verlangsamung des Durchmesserzwachses im unteren Teil der Stämme. Am unteren Ende der Krone ist diese Erscheinung weniger bemerkbar. Allein die höchsten Prozentsätze von 60 p. 100 und 65 p. 100 wirken sich durch eine allgemeine Erschlaffung des Stammes aus.

- Der Volumenzuwachs wird schwach von der Ástung beeinträchtigt. Nur die Prozentsałze von 60 p. 100 und 65 p. 100 führen zu einem merklichen Ertragsverlust. Dieser Verlust wird übrigens teilweise beim Schälen und Sägen ausgeglichen, und zwar dank der besseren, fast zylindrischen Form der Stämme.

- Bei Standortverhältnissen, die mit denen unseres Versuches zu vergleichen wären, Iässt sich wohl empfehlen, einen Prozentsatz von 50 p. 100 bis 55 p. 100 der Gesamthahe der Bäume für die Astung zu wählen. Die Ástung soll im frühen Alter beginnen und dann die Entwicklung der Baume regelmässig begleiten. Wenn es sich um I 214 handelt, hat sich die Ästung darum zu kümmern, die Gabelungen zu entfernen und schon in den ersten Jahren nach der Anpflanzung zu einer gut gediegenen Krone zu verhelfen.

- Später sina Untersuchungen anzustellen über den Einfluss der Ästung auf die Zugholzbildung und eine eventuelle Farbkernausbildung.

\section{Références bibliographiques}

BONDUELLE P., 1971. Etude théorique et pratique de l'élagage du peuplier $\alpha$ I $214 \%$. Rapport annuel de l'AFOCEL, 293-345.

Van BUGGENHOUDT M., 1954. Recherches sur les fibres gélatineuses. Publication Union Allumettiẻre Overboelare, Grammont, Belgique.

DEVEAUX C., 1969. Quelques considèrations sur l'ẻlagage du peuplier. Revue forestière Françoise, no 4, juillet-août, 281-284.

FRISON G., 1975 ? Ricerche sulla potatura di allevamento in pioppeto nel periodo estivo. Istifuto di sperimentozione per la pioppicoltura, Casale Monferrato.

POLGE H., 1967. Premiers résultats de l'expérience d'élagage de branche vivantes d'Epinal. Revue Forestière Française, n० 8-9, aoot-septembre, 530-546.

POLGE H., 1969. Densité de plantation et élagage de branches vivantes, ou pourquoi, quand et comment élaguer? Revue Forestière Fronçaise, $n^{\circ}$ spécial \& Progrès techniques en Sylviculture $\$$.

POLGE H., KELLER R., THIERCELIN F., 1973. Influence de l'élagage de branches vivantes sur la structure des accroissements annuels et sur quelques caractéristiques du bois de douglas et de grandis. Annales des Sciences Forestières, 30 (2), 127-140.

POURTET J., 1961. La culture du peuplier. J. B. Baillière et Fils, Paris, 2e édition.

SACHSSE H., 1965. Untersuchungen uber den Einfluss der Ästung auf die Farbkern $=$ und Zugholausbildung einiger Pappelsorten. Holz als Roh-Werkstoff, 23 (11), 425-34.

SACRE E., 1974? Proportion de faux ccur et de bois de tension dans les peupliers \& Robusta élagués et non élagués. Station de Technologie Forestière, Gembloux. 